\title{
SINGULARITIES IN THE NILPOTENT SCHEME OF A CLASSICAL GROUP
}

\author{
BY \\ WIM HESSELINK
}

\begin{abstract}
If $(X, x)$ is a pointed scheme over a ring $k$, we introduce a (generalized) partition $\operatorname{ord}(x, X / k)$. If $G$ is a reductive group scheme over $k$, the existence of a nilpotent subscheme $N(G)$ of $\operatorname{Lie}(G)$ is discussed. We prove that $\operatorname{ord}(x, N(G) / k)$ characterizes the orbits in $N(G)$, their codimension and their adjacency structure, provided that $G$ is $G l_{n}$, or $S p_{n}$ and $1 / 2 \in k$. For $S O_{n}$ only partial results are obtained. We give presentations of some singularities of $N(G)$. Tables for its orbit structure are added.
\end{abstract}

Introduction. Let $G$ be a reductive algebraic group over a field of characteristic $p$. Let $g$ be its Lie-algebra and $N(G)$ the closed subset of the nilpotent elements of $\mathrm{g}, \mathrm{cf}$. [19]. The $G$-orbits in $N(G)$ are characterized by weighted Dynkin diagrams, cf. [20, III]. Consider the following question. Is it possible to classify the orbits in $N(G)$ using only the local structure of the variety $N(G)$ ? We prove in (4.3) that the answer is positive if $G$ is $G l_{n}$ or if $G$ is $S p_{n}$ and $p$ $\neq 2$.

To this end we introduce a local invariant "ord" for any pointed scheme in $\S 1$. We develop the theory of $N(G)$ over an arbitrary ground ring $k$ in $\S 2$. In $\S 3$ we restrict our attention to the classical group schemes. Using a cross section we obtain information about the orbit structure of $N(G)$. Our main theorem (4.2) relates ord $(x, N(G) / k)$ to the Jordan normal form of the nilpotent endomorphism induced by $x$ in the classical representation.

This paper is a condensed version of [13]. The author wishes to express his gratitude to his thesis adviser, Professor T. A. Springer.

Conventions and notations. The cardinality of a set $V$ is denoted by $\# V$ Any infinite cardinal is represented by $\infty$. If $x$ is a real number then $[x]$ is the greatest integer in $x$. All rings are commutative with 1 . Let $M$ be a module over a ring $A$. If $M$ is free the rank of $M$ is denoted by $\operatorname{rg}_{A} M$. An element $c \in$ $A$ is called $M$-regular if $a: M \rightarrow M$ is injective. Let $\mathrm{a}=\left(a_{1}, \ldots, a_{r}\right)$ be a

Received by the editors March 18, 1975.

AMS (MOS) subject classifications (1970). Primary 14B05, 14L15; Secondary 05A17, 10C30, $13 \mathrm{H15}, 20 \mathrm{G} 35$.

Key words and phrases. Partitions, linear extension of a local ring, regular se juence, classical group scheme, orbit, cross section, nilpotent variety, Kleirian singularity 
sequence in $A$. The ideal generated by a is denoted by $\langle a\rangle$. The sequence is called $M$-regular if $a_{i}$ is $\left(M /\left\langle\left(a_{j}\right)_{j<i}\right\rangle M\right)$-regular for all $i$, cf. [12, $\left.0_{\mathrm{IV}} 15.1\right]$.

Unless stated otherwise $k$ is an arbitrary ground ring. General references for schemes and group schernes are [11], [12] and [8]. If we consider a $k$-scheme as a functor from $k$-algebras to sets, $\mathrm{cf}$. [11, p. 17], then the letter $R$ is used to denote an arbitrary $k$-algebra. If $X$ is a $k$-scheme and $R$ is a $k$-algebra then $X_{(R)}$ is the $R$-scheme $X \otimes_{k} R$. If $X$ is an affine scheme then its coordinate ring is denoted by $A(X)$. If $A$ is a local ring its maximal ideal is denoted by $\mathrm{nt}_{A}$ and its residue field by $\mathrm{k}(A)$. If $X$ is a scheme and $x \in X$ then we write $\mathrm{m}_{x}:=\mathfrak{m}_{A}$ and $\mathrm{k}(x):=\mathrm{k}(A)$ where $A:=0_{X, x}$.

1. A near-partition for a local $k$-algebra.

(1.1) A near-partition $\lambda$ is a subset of $\mathrm{N}^{2}$ such that if $(m, n) \in \lambda$ and $i \leqslant$ $m$ and $j \leqslant n$ then $(i, j) \in \lambda$. The set of near-partitions is denoted by NP. The duality mapping $D: N P \rightarrow N P$ is induced by $(i, j) \mapsto(j, i)$. The set NP is ordered by $\lambda \leqslant \mu$ if and only if $\lambda \subset \mu$. We write $|\lambda|:=\# \lambda$. A near-partition $\lambda$ is called a partition if $|\lambda|<\infty$. The set of partitions is denoted by $P$.

If $\lambda \in N P$, the nonincreasing sequences $\lambda^{*}$ and $\lambda_{*}$ in $\{0\} \cup N \cup\{\infty\}$ are defined by

$$
\lambda^{n} \geqslant i \Leftrightarrow(n, i) \in \lambda \Leftrightarrow \lambda_{i} \geqslant n
$$

Clearly $\lambda_{i}=(D \lambda)^{i}=\sup \left\{n \in \mathbf{N} \mid \lambda^{n} \geqslant i\right\}$, and dually. A near-partition $\lambda$ is completely determined by its sequence $\lambda^{*}$ (or $\lambda_{*}$ ). We write $\lambda_{*}=\left(\lambda_{1}, \ldots\right.$, $\left.\lambda_{r}\right)$ if $\lambda_{i}=0$ for $i>r$. If $\lambda, \mu \in N P$, we define $\lambda+\mu \in N P$ by $(\lambda+\mu)^{n}:=$ $\lambda^{n}+\mu^{n}$, where $x+\infty:=\infty+x:=\infty$ for all $x$. If $\lambda_{*}=\left(\lambda_{1}, \ldots, \lambda_{r}\right)$ and $\mu_{*}=\left(\mu_{1}, \ldots, \mu_{s}\right)$ then $(\lambda+\mu)_{*}$ is the sequence obtained by ordering $\left(\lambda_{1}, \ldots\right.$, $\left.\lambda_{r}, \mu_{1}, \ldots, \mu_{s}\right)$, see [9, Proposition 6].

(1.2) Definition. A linear extension over a ring $k$ is a surjective morphism $\epsilon: E \rightarrow A$ of local $k$-algebras such that $\mathfrak{m}_{E} \operatorname{ker}(\epsilon)=0$. Its near-partition $\operatorname{ord}(\epsilon)$ is defined by

$$
\operatorname{ord}^{n}(\epsilon):=\operatorname{rg}_{k(E)}\left(\operatorname{ker}(\epsilon) \cap m_{E}^{n+1}\right)
$$

A linear extension $\epsilon: E \rightarrow A$ is called versal over $k$ if for any linear extension $\zeta: F \rightarrow B$ over $k$ and any local $k$-morphism $\phi: A \rightarrow B$ there exists a (clearly local) $k$-morphism $\gamma: E \rightarrow F$ with $\zeta \circ \gamma=\phi \circ \epsilon$, see diagram (i).

(1.3) Proposition. Let diagram (i) be a commutative diagram of $k$ algebras such that $\epsilon$ and $\zeta$ are linear extensions, that $\phi$ is a flat local morphism and that $\mathrm{m}_{A} B=\mathrm{m}_{B}$. Then we have $\operatorname{ord}(\epsilon) \geqslant \operatorname{ord}(\zeta)$. 
(i)

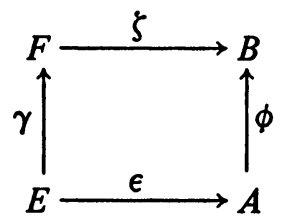

Proof. Let $n \in \mathrm{N}$. We prove that $\operatorname{ord}^{n}(\epsilon) \geqslant \operatorname{ord}^{n}(\zeta)$. It suffices to prove that the ideal $\operatorname{ker}(\zeta) \cap m_{F}^{n+1}$ is generated by the image of $\operatorname{ker}(\epsilon) \cap m_{E}^{n+1}$. We may assume that $\operatorname{ker}(\epsilon) \cap \mathrm{m}_{E}^{n+1}=0$. Now the mapping $\mathrm{m}_{E}^{n+1} \rightarrow A$ induced by $\epsilon$ is an injection of $A$-modules. Since $B$ is flat over $A$, it follows that $\mathrm{m}_{E}^{n+1} \otimes_{E} B \rightarrow B$ is injective and hence that $\operatorname{Tor}^{E}\left(E / \mathrm{m}_{E}^{n+1}, B\right)=0$. This implies injectivity of

$$
\operatorname{ker}(\zeta) \otimes_{E}\left(E / m_{E}^{n+1}\right) \rightarrow F \otimes_{E}\left(E / m_{E}^{n+1}\right)
$$

so that $\operatorname{ker}(\zeta) \cap \mathfrak{m}_{E}^{n+1} F=\mathfrak{m}_{E}^{n+1} \operatorname{ker}(\zeta)=0$. On the other hand $\mathfrak{m}_{A} B=\mathfrak{m}_{B}$ implies that $\mathrm{m}_{E} F+\operatorname{ker}(\zeta)=\mathrm{m}_{F}$, so that $\mathrm{m}_{E}^{n+1} F=\mathrm{m}_{F}^{n+1}$. This proves $\operatorname{ker}(\zeta) \cap \mathrm{m}_{F}^{n+1}=0$.

(1.4) Let $A$ be a local $k$-algebra. If $\epsilon: E \rightarrow A$ is a versal linear extension over $k$ then (1.3) implies that $\operatorname{ord}(\epsilon) \geqslant \operatorname{ord}(\zeta)$ for any linear extension $\zeta: F \rightarrow A$ over $k$. On the other hand there exists a versal linear extension $\epsilon: E \rightarrow A$ over $k$. In fact, write $A=R / A$ where $R$ is some polynomial $k$-algebra. Let $M$ be the ideal in $R$ such that $\mathfrak{m}_{A}=M / J$. Then $R / M J \rightarrow A$ is a versal linear extension over $k$, compare [15, p. 37]. Now we can give the following:

Definition. $\operatorname{ord}(A / k):=\operatorname{ord}(\epsilon)$ where $\epsilon: E \rightarrow A$ is some (or any) versal linear extension over $k$.

Example. Let $k$ be a field. Put $H:=k\left[T_{1}, \ldots, T_{m}\right]$. Let $\mathrm{a}=\left(a_{1}\right.$, $\left.\ldots, a_{r}\right)$ be a sequence in $H$. Let $a_{i}$ be homogeneous of degree $1+\lambda_{i}$ where $\lambda$ is a partition with $\lambda_{r+1}=0$. Assume that the ideal $\langle a\rangle$ is not generated by a strict subsequence of a. Consider the local ring $A:=(H /\langle\mathrm{a}\rangle)_{\mathfrak{p}}$ where $\mathfrak{p}=\left\langle T_{1}\right.$, $\left.\ldots, T_{m}\right\rangle$. Then $\operatorname{ord}(A / k)=\lambda$.

In fact $\epsilon:(H / p\langle a\rangle)_{\mathfrak{p}} \rightarrow A$ is a versal linear extension over $k$ and $\operatorname{ord}^{n}(\epsilon)$ $=\#\left\{i \mid \lambda_{i} \geqslant n\right\}=\lambda^{n}$.

(1.5) Proposition. Let $A$ be a local $k$-algebra and $R$ a $k$-algebra. Assume that $A$ or $R$ is flat over $k$. Let $\mathfrak{p} \in \operatorname{Spec}\left(A \otimes_{k} R\right)$ contract to $\mathrm{m}_{A}$. Then $\operatorname{ord}(A / k) \leqslant \operatorname{ord}\left(\left(A \otimes_{k} R\right)_{p} / R\right)$.

Proof. Let $\epsilon: E \rightarrow A$ be a versal linear extension over $k$. Put $I:=$ $\operatorname{ker}(\epsilon)$. Let $q \in \operatorname{Spec}(E \otimes R)$ be the inverse image of $\mathfrak{p}$. Since $A$ or $R$ is flat over $k,(I \otimes R)_{\mathrm{q}}$ is an ideal in $(E \otimes R)_{\mathrm{q}}$. Put $F:=(E \otimes R)_{\mathrm{q}} / \mathrm{q}(I \otimes R)_{\mathrm{q}}$, so that $\zeta: F \rightarrow(A \otimes R)_{\mathfrak{p}}$ is a linear extension over $R$. One verifies that $I \otimes_{\mathrm{k}(E)} \mathrm{k}(F) \rightarrow \operatorname{ker}(\zeta)$ is injective and hence that ord $(\epsilon) \leqslant \operatorname{ord}(\zeta)$. This suffices. 
(1.6) Proposition. Let $A$ be a local $k$-algebra, $\mathrm{x}=\left(x_{1}, \ldots, x_{m}\right)$ an $A$-regular sequence in $\mathrm{m}_{A}$ and $f$ a nonzero element of $\langle\mathrm{x}\rangle$. Put $B=A /\langle f\rangle$ and $C=A /\langle\mathrm{x}\rangle$. Let $r \in \mathrm{N}$ and let $\rho$ be a partition with $\rho_{*}=(r-1)$.

(a) If $f \in \mathrm{m}_{A}^{r}$ then $\rho+\operatorname{ord}(A / k) \leqslant \operatorname{ord}(B / k)$.

(b) If $f \notin \mathrm{m}_{A}^{r+1}$ then $\operatorname{ord}(B / k) \leqslant \rho+\operatorname{ord}(C / k)$.

Proof. Let $\epsilon: E \rightarrow A$ be a versal linear extension over $k$. Put $I=$ $\operatorname{ker}(\epsilon)$. Choose $y_{i} \in E$ with $\epsilon\left(y_{i}\right)=x_{i}$ and $g \in E$ with $\epsilon(g)=f$. Put $F:=$ $E / g m_{E}$ and $G:=E / m_{E}\langle y\rangle$. The linear extensions $\zeta: F \rightarrow B$ and $\eta: G \rightarrow C$ are versal over $k$. Since $\mathbf{x}$ is a regular sequence, we have $I \cap\langle y\rangle=0$. So the induced mappings $I \rightarrow \operatorname{ker}(\zeta)$ and $I \rightarrow \operatorname{ker}(\eta)$ are injective. This implies that $\operatorname{ord}(\epsilon) \leqslant \operatorname{ord}(\zeta)$ and $\operatorname{ord}(\epsilon) \leqslant \operatorname{ord}(\eta)$.

(a) Now it suffices to prove:

(*) If $n<r$ then $1+\operatorname{ord}^{n}(\epsilon)=\operatorname{ord}^{n}(\zeta)$.

We may assume that $g \in \mathrm{m}_{E}^{n+1}$. The cokernel of the injection $I \cap \mathrm{m}_{E}^{n+1} \rightarrow$ $\operatorname{ker}(\zeta) \cap \mathrm{m}_{F}^{n+1}$ is isomorphic to $\langle g\rangle / g \mathrm{~m}_{E}$; this proves (*).

(b) By (*) it suffices to prove: If $f \notin m_{A}^{n+1}$ then $\operatorname{ord}^{n}(\zeta) \leqslant \operatorname{ord}^{n}(\eta)$. We may assume $g \in\langle y\rangle$. Since $f \notin \mathrm{m}_{A}^{n+1}$ we have $g \notin \mathfrak{m}_{E}^{n+1}$. Using that $I \cap\langle y\rangle=$ 0 , one shows that the mapping $\operatorname{ker}(\zeta) \cap \mathrm{m}_{F}^{n+1} \rightarrow \operatorname{ker}(\eta) \cap \mathrm{m}_{G}^{n+1}$ is injective.

REMARK. Usually (1.6) (a) is applied in the situation where $f$ itself is $A$ regular, $m=1$ and $x_{1}=f$.

(1.7) If $X$ is a $k$-scheme and $x \in X$ then $(X, x)$ is called a pointed $k$-scheme. We define $\operatorname{ord}(x, X / k):=\operatorname{ord}\left(O_{X, x} / k\right)$. Pointed $k$-schemes $(X, x)$ and $(Y, y)$ are called smoothly equivalent if there are smooth $k$-morphisms $f: Z \rightarrow X, g$ : $Z \rightarrow Y$ and a point $z \in Z$ with $f(z)=x, g(z)=y$. This is an equivalence relation on the class of pointed $k$-schemes, to be denoted by $(X, x) \sim(Y, y)$. See [12, IV 17] for the definition and the basic properties of smooth morphisms.

THEOREM. If $(X, x) \sim(Y, y)$ then $\operatorname{ord}(x, X / k)=\operatorname{ord}(y, Y / k)$.

Proof. We may assume that there is a smooth $k$-morphism $f: X \rightarrow Y$ with $f(x)=y$.

Using the regularity of the noetherian local ring $O_{X, x} / m_{y} O_{X, x}$ and the arguments of the proof of [12, IV 19.2.9], we construct a subscheme $Z$ of $X$ containing $x$ such that $O_{Z, x}=0_{X, x} /\langle a\rangle$ where a is an $O_{X, x}$-regular sequence, that $\mathrm{O}_{Y, y} \rightarrow \mathrm{O}_{Z, x}$ is flat and that $\mathrm{m}_{y} \mathrm{O}_{Z, x}$ is the maximal ideal of $\mathrm{O}_{Z, x}$. By (1.6) (a) we have ord $(x, X / k) \leqslant \operatorname{ord}(x, Z / k)$. Using (1.3) one proves that $\operatorname{ord}(x, Z / k) \leqslant \operatorname{ord}(y, Y / k)$.

We may assume that $Y=\operatorname{Spec} A$ and $y=\mathrm{m}_{A}$ where $A$ is a local $k$-algebrd. Choose a versal linear extension $\epsilon: E \rightarrow A$ over $k$. By [12, IV 18.1.1] there is a smooth $E$-algebra $R$ such that $\operatorname{Spec}\left(A \otimes_{E} R\right)$ is isomorphic to an open neigh- 
bourhood of $x$ in $X$. So $\mathcal{O}_{X, x} \cong\left(A \otimes_{E} R\right)_{p}$ for some $\mathfrak{p} \in \operatorname{Spec}\left(A \otimes_{E} R\right)$ contracting to $\mathrm{m}_{A}$. By $(1.5)$ we have $\operatorname{ord}(A / E) \leqslant \operatorname{ord}\left(\left(A \otimes_{E} R\right)_{\mathfrak{b}} / R\right)$. It is easy to see that this implies ord $(y, Y / k) \leqslant \operatorname{ord}(x, X / k)$.

(1.8) The following remark will not be used in the sequel. For proofs and details we refer to [13].

REMARK. Let $A$ be a noetherian local $k$-algebra. Then $\operatorname{ord}(A / k)$ is a partition. It is equal to $\operatorname{ord}(\hat{A} / k)$ where $\hat{A}$ is the completion of $A$. If $k$ is noetherian regular and $A$ is of essentially finite type over $k$, then $\operatorname{ord}(A / k)=\operatorname{ord}(A / \mathrm{Z})$. $A$ is regular if and only if $\operatorname{ord}(A / Z)=0$. If $A=R / J$ where $J$ is an ideal in a noetherian regular local ring $R$, then $\operatorname{ord}(A / Z)$ is determined by the sequence $v_{*}(J), \mathrm{cf} .[14$, p. 209].

2. The nilpotent scheme.

(2.1) Consider an action $h$ of an affine group scheme $G$ on an affine scheme $X$ over $k$. We have the morphisms $h, \mathrm{pr}_{2}: G \times_{k} X \rightrightarrows X$. The orbit $G x$ of $x \in X$ is defined as the subset $h\left(\operatorname{pr}_{2}^{-1}(x)\right)$ of $X$. Let $V$ be a subscheme of $X$. Let $U$ be the open set where the induced morphism $h^{V}: G \times{ }_{k} V \rightarrow X$ is smooth. $V$ is called a cross section at $x$ if $x \in V$ and $e_{(V)}(x) \in U$. Here $e_{(V)}$ : $V \rightarrow G \times{ }_{k} V$ is induced by the unit $e \in G(k)$. The subscheme $V$ is called a global cross section if $U \rightarrow \operatorname{Spec}(k)$ is surjective. $V$ is called an invariant subscheme if the morphism $h^{V}$ factorizes over $V$.

Let $A(X)^{G}$ be the equalizer of the comorphisms $A(X) \rightrightarrows A(G) \otimes_{k} A(X)$. If $Y$ is an affine $k$-scheme, a $G$-invariant $k$-morphism $f: X \rightarrow Y$ corresponds to a comorphism $A(Y) \rightarrow A(X)$ factorizing over $A(X)^{G}$. We define the affine quotient of the action by $[X / G]:=\operatorname{Spec}\left(A(X)^{G}\right)$. It is called universal if the induced morphism $\left[X_{(R)} / G_{(R)}\right] \rightarrow[X / G]_{(R)}$ is an isomorphism for any $k$-algebra $R$.

REMARKS. (a) Let $G$ be smooth over $k$. Then $\operatorname{pr}_{2}$ and $h$ are smooth morphisms. If $x^{\prime} \in G x$ then $(X, x) \sim\left(X, x^{\prime}\right)$, cf. (1.7). If $V$ is a cross section at $x$ then $(X, x) \sim(V, x)$.

(b) The condition, that the affine quotient $[X / G]$ is universal, is a local condition on $\operatorname{Spec}(k)$ for the topology $(f p q c)$, cf. [8, IV], see [13, p. 38]. If $k$ is a field any affine quotient is universal.

(c) Other types of quotients are discussed in [17, p. 3].

(2.2) Proposition. Assume in (2.1) that the morphism $X \rightarrow \operatorname{Spec}(k)$ is smooth and irreducible cf. [12, IV 4.5.5], and that $V$ is affine and a global cross section.

(a) The morphism $A(X)^{G} \rightarrow A(V)$ is injective.

(b) If $A(X)^{G} \rightarrow A(V)$ is bijective then $[X / G]$ is universal. 
Proof. (a) Consider a nonzero $f \in A(X)^{G}$. Assume that $f \mid V=0$. There is a commutative diagram (i), so we have $f \circ h^{V}=0$.

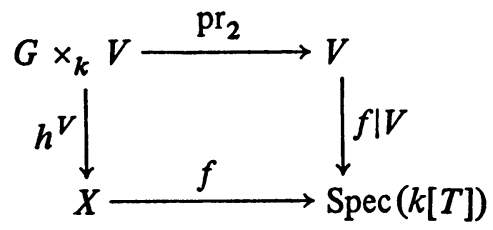

The morphism $h^{V}$ is flat on $U$, so $h^{V}(U)$ is an open subset of $X$ with $f h^{V}(U)$ $=0$. Since $f \neq 0$, there is a generic point $x$ of $\operatorname{Supp}\left(f O_{X}\right)$. Let $\mathfrak{p} \in \operatorname{Spec}(k)$ be the image of $x$. Let $\xi$ be the unique generic point of $X \otimes_{k} k(p)$. As $h^{V}(U)$ $\rightarrow \operatorname{Spec}(k)$ is surjective we have $\xi \in h^{V}(U)$ and hence $x \neq \xi$. Since $0_{X, x} \otimes_{k}$ $\mathrm{k}(\mathfrak{p})$ is regular there is an $O_{X, x} \otimes_{k} \mathrm{k}(\mathfrak{p})$-regular element $t \in \mathrm{m}_{x}$. By [12, IV 11.3.7], $t$ is $O_{X, x}$-regular. It is easy to see that this contradicts the choice of $x$. The argument used here was suggested by $P$. Deligne.

(b) Let $R$ be a $k$-algebra. We have to prove that $u: A(X)^{G} \otimes R \rightarrow$ $A\left(X_{(R)}\right)^{G}(R)$ is bijective. As the assumptions of (a) are stable under base-change, the morphism $v: A\left(X_{(R)}\right)^{G}(R) \rightarrow A(V) \otimes R$ is injective by (a). So it suffices to observe that $v \circ u$ is bijective.

(2.3) Let $G$ be a smooth affine group scheme over $k$. Recall that the Liealgebra $\operatorname{Lie}(G)$ is defined as the group functor such that $\operatorname{Lie}(G)(R)$ is the (additively written) kernel of the morphism $G\left(R[\delta] /\left\langle\delta^{2}\right\rangle\right) \rightarrow G(R)$ induced by $\delta \mapsto$ 0 where $R$ is a $k$-algebra. $\operatorname{Lie}(G)$ is a smooth affine group scheme, in fact a vector bundle. There is a canonical action of $G$ on $\operatorname{Lie}(G)$. If $R$ is a $k$-algebra then $\operatorname{Lie}(G)_{(R)}=\operatorname{Lie}\left(G_{(R)}\right)$. See [8, II 4]. Usually we write $8:=\operatorname{Lie}(G)$.

If $K$ is a field over $k$, a section $x \in g(K)=\operatorname{Lie}\left(G_{(K)}\right)(K)$ is nilpotent if and only if its image is a nilpotent endomorphism of $F$ for some (or any) immersion of $G_{(K)}$ in a $K$-group $G l(F)$, cf. [2, p. 151]. A point $x \in \mathfrak{g}$ is called nilpotent if the corresponding section $x \in \mathfrak{g}(\mathrm{k}(x))$ is nilpotent.

(2.4) Definition. Let $G$ be a reductive group scheme over $k$, cf. [8, XIX 2.7]. If the affine quotient $[g / G]$ is universal, cf. (2.1), then we define the nilpotent scheme $N(G):=p^{-1} p(0)$ where $0 \in \mathfrak{g}(k)$ is the zero section and $p: g \rightarrow$ $[g / G]$ is the quotient morphism.

Proposition. Let $N(G)$ be defined.

(a) $N(G)$ is a G-invariant closed subscheme of $\mathbf{g}$.

(b) If $R$ is a k-algebra then $N\left(G_{(R)}\right)=N(G)_{(R)}$.

(c) $A$ point $x \in \mathfrak{g}$ is nilpotent if and only if $x \in N(G)$.

Proof. (a) is trivial. (b) is a consequence of the assumption that $[g / G]$ is universal. (c) By (b) we may assume that $k$ is a field and that $x \in g(k)$. Now it is well known. The "if-part" follows from Cayley-Hamilton by an embedding 
of $G$ in some $G l(F)$. The "only-if-part" is a consequence of the following

LEMMA. Let $G$ be a reductive $k$-group over a field $k$. If $x \in g(k)$ has the additive Jordan decomposition $x=x_{s}+x_{n}$, then $x_{s}$ is in the closure of the orbit $G x$.

Proof. Adapt [22, (4.4)] or [21, p. 92].

(2.5) Let $G$ be a reductive group scheme over $k$. By $(2.1)(b)$ the existence of a nilpotent scheme is a local condition on $\operatorname{Spec}(k)$ for the topology $(f p q c)$. So we assume that $G$ is of constant type (cf. [8, XXII 2.7]) with specified root system $R=(M, R, \rho)$, i.e. a root system $R$ in a given lattice $M$ (cf. [7, p. 287]). Let $t$ be the torsion index (cf. [7, p. 294]). Let $f$ be the connection index (cf. $[4$, p. 167]). Consider the following conditions:

(i) $t^{-1} \in k$ and if $R \cap 2 M \neq \varnothing$ then $1 / 2 \in k$, cf. [7, p. 296].

(ii) $t^{-1} f^{-1} \in k$.

(iii) If $R$ has a component of type $A_{l}$ then $(l+1)^{-1} \in k$, of type $B_{l}, C_{l}$, $D_{l}, G_{2}$ then $1 / 2 \in k$, of type $E_{6}, E_{7}, F_{4}$ then $1 / 6 \in k$, of type $E_{8}$ then $1 / 30$ $\in k$.

The conditions (ii) and (iii) are equivalent and imply (i).

(2.6) THEOREM. Let $G$ be as in (2.5) satisfying condition (i).

(a) The affine quotient $[\mathrm{g} / G]$ is universal. The quotient morphisms $p: g \rightarrow[\mathrm{g} / G]$ is flat. $N(G)$ is defined and flat over $k$.

(b) Let $T$ be a maximal torus of $G$ with Weyl group $W$, cf. [8, XXII 3]. Put $\mathrm{t}:=\mathrm{Lie}(T)$. The affine quotient $[\mathrm{t} / W]$ is universal. The canonical morphism $[\mathrm{t} / W] \rightarrow[\mathrm{g} / G]$ is an isomorphism.

(c) Assume that (2.5) (ii) holds. Let $\pi: G \rightarrow \operatorname{ad}(G)$ be the projection onto the adjoint group, $c f .[8, \mathrm{XXII} 4.3]$. Then $N(\operatorname{ad}(G))$ is defined and equal to $N(G)$.

Proof. (1) We may assume that $G$ is split with respect to a (resp. the) maximal torus $T$, cf. [8, XXII 2.3]. Now $T=D_{S}(M)$ and $A(\mathrm{t})=S(M) \otimes k$. The group scheme $W$ is the constant group scheme associated to the abstract Weyl group of $R$. By (2.5) (i) and [7, pp. 295, 296] the affine quotient $[t / W]$ is universal and the quotient morphism $t \rightarrow[t / W]$ is flat.

(2) By [8, XIII 5.1] and [12, IV 17.8.3] the subscheme $t$ is a global cross section for the action of $G$ on $\mathfrak{g}$, cf. (2.1). By (2.2) this implies that $A(\mathfrak{g})^{G} \rightarrow$ $A(\mathrm{t})^{W}$ is injective.

(3) We may assume that $k=\mathrm{Z}[1 / m]$, $\mathrm{cf}$. [8, XXV 1]. It follows from $\left[20\right.$, II 3.17'] and [22, p. 220] that $A(g)^{G} \otimes_{k} \mathrm{Q} \rightarrow A(\mathrm{t})^{W} \otimes_{k} \mathrm{Q}$ is bijective. Consider $a \in A(\mathrm{t})^{W}$. There is $a_{1} \in A(\mathrm{~g})^{G}$ and a nonzero $s \in k$ with $a_{1} \mid \mathrm{t}=s a$. Put $R=k /\langle s\rangle$. Now $a_{1} \otimes 1_{R} \mid \mathrm{t}_{(R)}=0$, so by (2) we have $a_{1} \otimes 1_{R}=0$ in 
$A(\mathrm{~g}) \otimes R$. So there is $a_{2} \in A(\mathrm{~g})$ with $a_{1}=s a_{2}$. Since $s$ is $A(G) \otimes A(\mathrm{~g})$-regular we have $a_{2} \in A(\mathrm{~g})^{G}$. Since $s$ is $A(\mathrm{t})$-regular we have $a=a_{2} \mid \mathrm{t}$. This proves that $A(\mathrm{~g})^{G} \rightarrow A(\mathrm{t})^{W}$ is bijective. So we have proved (b).

(4) With (b) and (2) one proves that $[\mathrm{g} / G]$ is universal in the same way as in (2.2) (b). Let $U$ be the open subset of $g$ where $p$ is flat. Since $t \rightarrow[g / G]$ is flat by (1) and (b), and $t \subset g$ is a regular immersion, we have $t \subset U$ by [12,

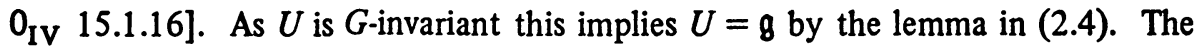
other assertions of (a) follow immediately.

(5) In the notations of [8, XXII], condition (2.5) (ii) implies that the central isogenies $G \rightarrow \operatorname{corad}(G) \otimes s s(G)$ and $s s(G) \rightarrow \operatorname{ad}(G)$ are étale morphisms, by $[8$, VIII 2.1] and $[8, \mathrm{XXI} 6.5]$. So we have an isomorphism

$$
A(\operatorname{Lie}(\operatorname{ad}(G)))^{\operatorname{ad}(G)} \otimes A(\operatorname{Lie}(\operatorname{corad}(G))) \cong A(\mathfrak{g})^{G} .
$$

With this isomorphism one proves (c).

REMARKS. (i) Assume that the order of the Weyl group is invertible in $k$. By $[22,(6.9)]$ the morphism $p$ is normal cf. [12, IV 6.8.1]. (ii) If $l \geqslant 2$ there is a semisimple group scheme $G$ of type $D_{l}$ over $\mathrm{Z}$ such that $[\mathrm{g} / G]$ is not universal.

(2.7) Corollary. Let $G$ be as in (2.6). Let $d_{1}, \ldots, d_{r}$ be the degrees of $R$. Consider the partition $\lambda$ defined by $\lambda_{i}:=d_{r+1-i}-1$ if $i \leqslant r$ and $\lambda_{r+1}$ $:=0$. Let $x$ be a point of the zero section of 8 . Then $\operatorname{ord}(x, N(G) / k)=\lambda$.

Proof. By (1.7) we may assume that $G$ is split with maximal torus $T$. Let $A(g)^{G}=k\left[a_{1}, \ldots, a_{r}\right]$ where $a_{1}, \ldots, a_{r}$ are algebraically independent and $a_{i}$ is homogeneous of degree $d_{r+1-i}=1+\lambda_{i}$, cf. [7, Theorem 3]. We have $O_{N(G), x}=O_{B, x} /\langle a\rangle$. Since $O_{B, x}$ is flat over $A(g)^{G}$ the sequence a is $O_{B, x}$-regular. By (1.6) (a) this implies that ord $(x, N(G) / k) \geqslant \lambda$. Let $p \in \operatorname{Spec}(k)$ be the image of $x$. By (1.5) we may replace $k$ by the residue field $k(p)$. Now the assertion follows from the example in (1.4).

REMARK. If $k$ is noetherian regular the multiplicity of the local ring $\mathcal{O}_{N(G), x}$ is equal to $\Pi_{i=1}^{r} d_{i}$, i.e. the order of the Weyl group. This is proved in $[13$, p. 55] using the methods of [18]. Compare [16, p. 386].

3. In the classical Lie-algebras.

(3.1) We fix a free $k$-module $F$ of rank $n$. The scheme $\operatorname{End}(F)$ is defined by $\operatorname{End}(F)(R):=\operatorname{End}_{R}\left(F \otimes_{k} R\right)$, cf. [11, I 9]. The group scheme $G l(F)$ (resp. $S l(F)$ ) is the open (resp. closed) subscheme of $\operatorname{End}(F)$ where the function det $\in A(\operatorname{End}(F)$ ) is invertible (resp. where det $=1) . G l(F)$ and $S l(F)$ are reductive group schemes over $k$ of type $A_{n-1}, \mathrm{cf}$. [6] and [8]. $\operatorname{End}(F)$ is identified with $\operatorname{Lie}(G l(F))$ by $x \leftrightarrow 1+\delta x$ where $x \in \operatorname{End}(F)(R)$, see (2.3) or [8, II 4]. Now $\operatorname{Lie}(\operatorname{Sl}(F))$ consists of the endomorphisms with zero trace. 
Assume $1 / 2 \in k$. Let $\epsilon$ be 0 or 1 . An $\epsilon$-form $\phi$ on $F$ is a nondegenerate bilinear form $\phi: F \times F \rightarrow k$ which is symmetric if $\epsilon=0$, alternating if $\epsilon=1$. By "nondegenerate" we mean that the mapping $F \rightarrow F^{\iota}$ defined by $f \mapsto$ $\phi(f,-)$ is bijective. Let $\phi$ be an $\epsilon$-form. The subgroup functor $G^{\prime}(F, \phi)$ of $G l(F)$ is defined by $x \in G^{\prime}(F, \phi)(R)$ if and only if

$$
\phi(x f, x g)=\phi(f, g) \quad(f, g \in F \otimes R) .
$$

We define $G(F, \phi):=G^{\prime}(F, \phi) \cap S l(F)$. If $\epsilon=0$ then $G(F, \phi)$ is the special orthogonal group scheme. If $\epsilon=1$ then $G(F, \phi)=G^{\prime}(F, \phi)$; it is the symplectic group scheme. Put $l:=[1 / 2 n]$ and $\zeta:=n-2 l$. So $\zeta$ is 0 or 1 . If $\epsilon=1$ then $\zeta=0$. Now $G(F, \phi)$ is a semisimple group scheme of type $B_{l}$ if $\epsilon=0, \zeta=1$, of type $C_{l}$ if $\epsilon=1, \xi=0$, of type $D_{l}$ if $\epsilon=\xi=0$, cf. [6] and [8]. The common Lie-algebra of $G(F, \phi)$ and $G^{\prime}(F, \phi)$ is denoted by $\mathfrak{g}(F, \phi)$. For $x \in$ End $(F)(R)$ we have $x \in \mathfrak{g}(F, \phi)(R)$ if and only if

$$
\phi(x f, g)+\phi(f, x g)=0 \quad(f, g \in F \otimes R) .
$$

Convention. In the rest of this paper we consider two cases.

Case I. $G:=G^{\prime}:=G l(F), l:=n$.

Case II. $(\epsilon, \zeta)$ where $\epsilon, \zeta \in\{0,1\}, \epsilon+\zeta \leqslant 1: 1 / 2 \in k, \phi$ is an $\epsilon$-form on $F$, $G:=G(F, \phi), G^{\prime}:=G^{\prime}(F, \phi), n=2 l+\zeta$.

In both cases $l$ is the reductive rank of $G$. We put $8:=\operatorname{Lie}(G)$. While considering Case II it is convenient to label concepts introduced for Case I with the index $I$, e.g. $\mathfrak{g} \subset g_{I}=\operatorname{End}(F)$.

(3.2) Lemma. Case II. Let $\phi_{1}$ be another $\epsilon$-form on $F$. Then there is a faithfully flat étale $k$-algebra $R$ such that $\phi_{1}$ and $\phi$ induce equivalent forms on $F \otimes R$.

Proof. By [15, pp. 34, 35] the scheme Isom $\left(\phi_{1}, \phi\right)$ is smooth over $k$. If $K$ is an algebraically closed field over $k$ then $\operatorname{Isom}\left(\phi_{1}, \phi\right)(K) \neq \varnothing$. Hence by [12, IV 17.16.3] there is a faithfully flat étale $k$-algebra $R$ with $\operatorname{Isom}\left(\phi_{1}, \phi\right)(R) \neq \varnothing$.

(3.3) Definition. In Case $I, z \in g(R)$ is called a standard nilpotent with base-data $(\mathrm{f}, \lambda)$ if $\mathrm{f}=\left(f_{1}, \ldots, f_{r}\right)$ is a sequence in $F \otimes R$ and $\lambda$ is a partition, such that $\lambda^{1}=r$, that the set $\left\{z^{a} f_{i}\right\}$, where $1 \leqslant i \leqslant r$ and $0 \leqslant a<\lambda_{i}$, is a basis of $F \otimes R$ and that $z^{a} f_{i}=0$ if $a \geqslant \lambda_{i}$.

In Case II, $z \in g(R)$ is called a standard nilpotent with base-data $(f, \lambda, \beta, \alpha)$ if $z \in g_{I}(R)$ is a standard nilpotent with base-data (f, $\lambda$ ), $\beta$ is a permutation of $\{1, \ldots, r\}$ where $r=\lambda^{1}$, and $\alpha:\{1, \ldots, r\} \rightarrow R$ is a mapping such that

$$
\left\{\begin{array}{l}
\phi\left(z^{a} f_{i}, z^{b} f_{j}\right)=(-1)^{a} \alpha(i) \text { if } j=\beta i \text { and } a+b+1=\lambda_{i} \\
\phi\left(z^{a} f_{i}, z^{b} f_{j}\right)=0 \text { otherwise. }
\end{array}\right.
$$


REMARK. Clearly $|\lambda|=n$. In Case II the assumptions imply

$$
\alpha(i)^{-1} \in R, \quad \beta^{2}=\mathrm{id}, \quad \lambda_{\beta i}=\lambda_{i}, \quad \alpha(\beta i)=(-1)^{\lambda_{i}-1+\epsilon} \alpha(i) .
$$

(3.4) The set $P_{\epsilon}$ is defined as the subset of $P$ consisting of the partitions $\lambda$ such that for any $m \geqslant 1$ with $m \equiv \epsilon(2)$ the number of indices $i$ with $\lambda_{i}=m$ is even. These partitions are called orthogonal, resp. symplectic; in [10, p. 556] We define $P(n)$ as the set of partitions $\lambda$ with $|\lambda|=n$, and $P_{\epsilon}(n):=P_{\epsilon} \cap P(n)$. We write $P_{(\epsilon)}$ to denote $P$ in Case I and $P_{\epsilon}$ in Case II. So in (3.3) we have $\lambda \in$ $P_{(\epsilon)}(n)$.

(3.5) If $x \in \mathrm{g}$ is nilpotent, cf. (2.3), then the section $x \in \mathrm{g}(\mathrm{k}(x))$ is a standard nilpotent by $[20, I V]$. Let $\lambda \in P(n)$. We define $D(\lambda)$ as the set of points $x \in \boldsymbol{g}$ such that the section $x$ is a standard nilpotent with partition $\lambda$. In case II we have $\mathscr{D}(\lambda)=\mathfrak{D}_{I}(\lambda) \cap \mathfrak{g}$, and $\mathscr{D}(\lambda) \neq \varnothing$ if and only if $\lambda \in P_{\epsilon}(n)$.

Let $k$ be a field and $x \in \mathscr{D}(\lambda)$. By [20, IV] we have $\mathscr{D}(\lambda)=G^{\prime} x$, and $\mathscr{D}(\lambda)$ $\neq G x$ if and only if we are in the very-even case: Case II $(0,0)$ with $\lambda_{i}$ even for all $i$.

(3.6) LEMmA. Case I. If $\lambda \in P(n)$, there is a standard nilpotent element $z \in g(k)$ with partition $\lambda$.

Case II. If $\lambda, \beta$ and $\alpha$ satisfy the conditions (3.3)(2), then there is an $\epsilon$ form $\phi_{1}$ on $F$ and a standard nilpotent element $z \in g\left(F, \phi_{1}\right)(k)$ with base-data (f, $\lambda, \beta, \alpha$ ) for some sequence $\mathbf{f}$ in $F$.

Proof. Case I is trivial.

Case II. Choose a standard nilpotent $z \in \mathfrak{g}_{I}(k)$ with base-data $(f, \lambda)$. Let $\phi_{1}: F \otimes F \rightarrow k$ be the bilinear form defined by (3.3)(1). One verifies that $\phi_{1}$ is an $\epsilon$-form on $F$ with $z \in g\left(F, \phi_{1}\right)(k)$.

(3.7) The standard cross section. Let $z \in g(k)$ be a standard nilpotent element with base-data (f, $\lambda$ ), resp. (f, $\lambda, \beta, \alpha$ ). Below we construct a linear subscheme $L \subset g$ such that $g(R)=[g(R), z] \oplus L(R)$ for any $k$-algebra $R$. This implies that the subscheme $z+L \subset g$ is a cross section for the adjoint action of $G$ in all points of the section $z$, cf. (2.1). In fact the tangent morphism of Ad: $G \times(z+L) \rightarrow g$ at the section $(e, z)$ is the surjective morphism $g \oplus L \rightarrow g$ given by $(x, y) \mapsto[x, z]+$ $y$. So smoothness of $\operatorname{Ad}$ at $(e, z)$ follows from [12, IV 17.11.1].

Let $\Psi$ be the set of pairs $(i, a)$ with $0 \leqslant a<\lambda_{i}$. Put $f(i, a):=z^{a} f_{i}$. Then $\{f(\psi) \mid \psi \in \Psi\}$ is a basis of $F$. Let $\{u(\psi)\} \overline{b e}$ the dual basis of $F$. This means that $\{u(\psi)\}$ is the basis of $F^{\sim}=\operatorname{Hom}(F, k)$ with

$$
\left\langle u(\psi), f\left(\psi^{\prime}\right)\right\rangle=\delta_{\psi, \psi^{\prime}} \quad \text { (Kronecker delta). }
$$

The coordinates $\xi\left(\psi ; \psi^{\prime}\right)$ on $g_{I}$ are defined by $\xi\left(\psi ; \psi^{\prime}\right)(x)=\left\langle u(\psi), x f\left(\psi^{\prime}\right)\right\rangle$. 
Clearly $\left\{\xi\left(\psi ; \psi^{\prime}\right) \mid \psi, \psi^{\prime} \in \Psi\right\}$ is a basis of $g_{I}(k)$. Let $\left\{e\left(\psi ; \psi^{\prime}\right)\right\}$ be the dual basis of $g_{I}(k)$. We have

$$
\begin{aligned}
e\left(\psi ; \psi^{\prime}\right) f\left(\psi^{\prime \prime}\right) & =\delta_{\psi^{\prime}, \psi^{\prime \prime} f(\psi),} \\
{[e(i, a ; j, b), z] } & =e(i, a ; j, b-1)-e(i, a+1 ; j, b)
\end{aligned}
$$

where $e(i, a ; j, b)=0$ if $a \geqslant \lambda_{i}$ or $b<0$. In Case I let $g_{i j}, L_{i j}$ and $L$ be the linear subschemes of $g$ defined by

$$
\begin{aligned}
\mathfrak{g}_{i j}(R) & :=\sum_{a, b} \operatorname{Re}(i, a ; j, b), \\
L_{i j}(R) & :=\sum_{R} \operatorname{Re}\left(i, a ; j, \lambda_{j}-1\right), \quad 0 \leqslant a<\min \left(\lambda_{i}, \lambda_{j}\right), \\
L(R) & :=\sum_{i, j} L_{i j}(R) .
\end{aligned}
$$

Then we have $g_{i j}=\left[g_{i j}, z\right] \oplus L_{i j}$ and $\mathfrak{g}=[\mathfrak{g}, z] \oplus L$.

Case II. The coordinates $\eta\left(\psi ; \psi^{\prime}\right)$ on $g$ are defined by $\eta\left(\psi ; \psi^{\prime}\right)(x)=$ $\phi\left(f(\psi), x f\left(\psi^{\prime}\right)\right)$. Since $\eta\left(\psi ; \psi^{\prime}\right)=(-1)^{1+\epsilon} \eta\left(\psi^{\prime} ; \psi\right)$ we have a basis of $g(k)^{-}$ consisting of the $\eta(i, a ; j, b)$ with $i<j$, or $i=j$ and $a<b+\epsilon$. Let $y\left(\psi ; \psi^{\prime}\right)$ be the dual basis of $g(k)$. One shows that

$$
\begin{aligned}
{[y(i, a ; j, b), z] } & =y(i, a ; j, b-1)+y(i, a-1 ; j, b) \\
& \text { if } i<j, \text { or } i=j \text { and } a<b-1, \\
{[y(i, a ; i, a+1), z] } & =y(i, a-1 ; i, a+1)+2 \epsilon y(i, a ; i, a), \\
{[y(i, a ; i, a), z] } & =y(i, a-1 ; i, a) \quad \text { if } \epsilon=1,
\end{aligned}
$$

where $y\left(\psi ; \psi^{\prime}\right)=0$ if not yet defined. For $i \leqslant j$ let $g_{i j}, L_{i j}$ and $L$ be the linear subschemes of $g$ defined by

$$
\begin{aligned}
\mathfrak{g}_{i j}(R) & :=\sum_{a, b} R y(i, a ; j, b), \\
L_{i j}(R) & :=\sum_{b} R y\left(i, \lambda_{i}-1 ; j, b\right) \quad \text { if } i<j, \\
L_{i i}(R) & :=\sum_{1} R y\left(i, \lambda_{i}-2+\epsilon-a ; i, \lambda_{i}-1-a\right), 0 \leqslant a \leqslant 1 / 2\left(\lambda_{i}-2+\epsilon\right), \\
L(R) & :=\sum_{i<j} L_{i j}(R) .
\end{aligned}
$$

Then we have $\mathfrak{g}_{i j}=\left[\mathfrak{g}_{i j}, z\right] \oplus L_{i j}$ and $\mathfrak{g}=[\mathfrak{g}, z] \oplus L$.

Let $F^{\curlyvee}$ be identified with $F$ in such a way that $\langle u, f\rangle=\phi(u, f)$. Putting $|i|_{a}=(-1)^{a} \alpha(i)^{-1}$, we get the following glossary: 


$$
\begin{aligned}
|i|_{a} f(i, a) & =u\left(\beta i, \lambda_{i}-1-a\right), \\
|i|_{a} \eta(i, a ; j, b) & =\xi\left(\beta i, \lambda_{i}-1-a ; j, b\right) \mid g, \\
y(i, a ; j, b) & =|i|_{a} e\left(\beta i, \lambda_{i}-1-a ; j, b\right)-(-1)^{\epsilon}|j|_{b} e\left(\beta j, \lambda_{j}-1-b ; i, a\right) \\
y(i, a ; i, a) & =|i|_{a} e\left(\beta i, \lambda_{i}-1-a ; i, a\right) \quad \text { if } i<j, \text { or } i=j \text { and } a<b, \\
y(i, a ; j, b) & =0 \quad \text { otherwise. }
\end{aligned}
$$

REMARK. In Case I our $z+L$ is one of the cross sections of Arnold [1].

(3.8) An elementary calculation shows that $L(k)$ is a free $k$-module of rank $l+\gamma_{(\epsilon)}(\lambda)$ if we write $\gamma_{(\epsilon)}(\lambda):=\gamma(\lambda)$ in Case I and $\gamma_{(\epsilon)}(\lambda):=\gamma_{\epsilon}(\lambda)$ in Case II where

$$
\begin{aligned}
& \gamma(\lambda):=2 \sum(i-1) \lambda_{i} \text { if } \lambda \in P(n), \\
& \gamma_{\epsilon}(\lambda):=\sum(i-1) \lambda_{i}+(2 \epsilon-1)\left[1 / 2 \#\left\{i \mid \lambda_{i} \equiv 1 \text { (2) }\right\}\right] \text { if } \lambda \in P_{\epsilon}(n) .
\end{aligned}
$$

Now the centralizer of $z$ in $g(k)$ is also a free $k$-module of rank $l+\gamma_{(\epsilon)}(\lambda)$. By $[20$, I 5.6] we have the following:

Corollary. Assume that $k$ is a field.

(a) If $x \in \mathcal{D}(\lambda)$ then $\operatorname{dim}(G x)=\operatorname{dim}(g)-l-\gamma_{(\epsilon)}(\lambda)$.

(b) There is a unique nilpotent orbit $C_{\mathrm{reg}}$ of maximal dimension.

$C_{\mathrm{reg}}=\mathfrak{O}(\nu)$ where $\nu_{*}=(n)$ in the Cases I and II $(\epsilon, 1-\epsilon)$ and $\nu_{*}=(\eta-$ $1,1)$ in Case II $(0,0)$. We have $\operatorname{dim}\left(C_{\mathrm{reg}}\right)=\operatorname{dim}(g)-l$. If $C$ is another nilpotent orbit in $g$ then $\operatorname{dim}(C) \leqslant \operatorname{dim}(g)-l-2$.

See also [1], [20, IV 2.28] and [21, p. 136].

(3.9) The mapping $\Sigma: P(n) \rightarrow P$ is defined by $(\Sigma \lambda)^{m}:=\Sigma_{i>m} \lambda_{i}(m \in$ $N)$. As the corresponding propositions in $[10$, p. 567] are false, we shall prove the following:

Proposition. Let $\lambda, \mu \in P_{(\epsilon)}(n)$ be such that

$$
\{\mu\}=\left\{\nu \in P_{(\epsilon)}(n) \mid \Sigma \lambda>\Sigma \nu \geqslant \Sigma \mu\right\}
$$

Then there are $\rho, \sigma, \tau \in P_{(\epsilon)}$ with $\lambda=\rho+\sigma, \mu=\rho+\tau$ and $\sigma, \tau$ as described in the following table. 


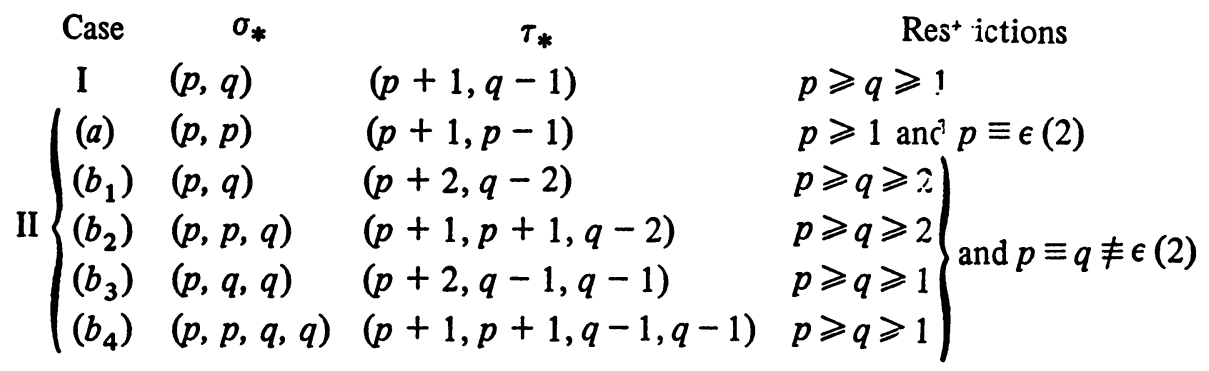

Proof. See (1.1) for the addition of partitions. Case I may be left to the reader. Case II. It is easy to see that we may assume disjointness: if $\lambda_{i}=$ $\mu_{j}$ then $\lambda_{i}=0$. Now we have to prove $\lambda=\sigma, \mu=\tau$ as in the table.

(a) Assume that there is a minimal $l \in \mathrm{N}$ with $\lambda_{l}$ with $\lambda_{l} \neq 0$ and $\lambda_{l} \equiv$ $\epsilon$ (2). There is a maximal $m \in N$ with $\lambda_{m}=\lambda_{l}$. Define $\nu \in P_{\epsilon}(n)$ by $\nu_{l}=$ $\lambda_{l}+1, \nu_{m}=\lambda_{m}-1$ and $\nu_{i}=\lambda_{i}$ otherwise. Clearly $\Sigma \lambda>\Sigma \nu$. Using disjointness one proves $\Sigma \nu \geqslant \Sigma \mu$, so that $\nu=\mu$ and, again by disjointness, we are in case (a).

(b) Now $\lambda_{i} \not \equiv \in(2)$ whenever $\lambda_{i}>0$. By disjointness there is an $m \in \mathbf{N}$ with $\mu_{m}>\lambda_{1}>\mu_{m+1}$. It is easy to see that we can define $\nu \in P_{\epsilon}(n)$ satisfying $\Sigma \nu>\Sigma \mu$ as follows:

If $\mu_{m} \not \equiv \epsilon$ (2), then $\nu_{m}=\mu_{m}-2$ and $\nu_{i}=\mu_{i}$ if $i<m$;

if $\mu_{m} \equiv \epsilon(2)$, then $\nu_{m-1}=\nu_{m}=\mu_{m}-1$ and $\nu_{i}=\mu_{i}$ if $i<m-1$;

if $\mu_{m+1} \neq \equiv \in(2)$, then $\nu_{m+1}=\mu_{m+1}+2$ and $\nu_{i}=\mu_{i}$ if $i>m+1$;

if $\mu_{m+1} \equiv \epsilon$ (2), then $\nu_{m+1}=\nu_{m+2}=\mu_{m+1}+1$ and $\nu_{i}=\mu_{i}$ if $i>m+2$.

One proves that $\Sigma \lambda \geqslant \Sigma \nu$, so that $\lambda=\nu$ and we are in one of the four cases (b).

(3.10) Theorem. Let $k$ be a field. Consider $z \in \mathfrak{D}(\lambda)$ and $x \in \mathfrak{D}(\mu)$. We have $z \in \overline{G x}-G x$ if and only if $\Sigma \lambda>\Sigma \mu$.

REMARK. This theorem is due to Gerstenhaber, see $[9$, p. 327] and [10, pp. 567-569]. His proof for Case II is incomplete, see (3.9). Our proof seems to be more explicit.

Proof. We may assume that $z$ and $x$ are rational points. So $z$ is a standard nilpotent in $g(k)$ with partition $\lambda$. If $i \in \mathrm{N}$ then the endomorphism $z^{i}$ of $F$ has rank $(\Sigma D \lambda)^{i}$, see (1.1) for the definition of $D$.

Assume that $z \in \overline{G x}-G x$. The rank of $z^{i}$ is less than or equal to the rank of $x^{i}$. This implies $\Sigma D \lambda \leqslant \Sigma D \mu$ and hence $\Sigma \lambda \geqslant \Sigma \mu$ by [9, p. 327]. As $\lambda \neq \mu$ it is easy to see that $\Sigma \lambda>\Sigma \mu$.

Assume that $\Sigma \lambda>\Sigma \mu$. We have to prove that $z \in \overline{G x}$. We may assume that $\lambda$ and $\mu$ are as in (3.9). So $\lambda$ and $\mu$ are not both very-even, cf. (3.5), and it suffices to prove that $z \in \overline{\mathscr{D}(\mu)}$. Using the notations of (3.7) we shall construct $y \in \mathfrak{g}(k)$ and a sequence $\mathrm{f}(t)(t \in k)$ in such a way that $z(t)=z+t y \in$ 
$g(k)$ is a standard nilpotent in $g_{I}(k)$ with base-data $(\mathrm{f}(t), \mu)$ if $t \neq 0$. This will prove $z \in \overline{D(\mu)}$.

Using a direct sum decomposition we may assume $\rho=0, \lambda=\sigma, \mu=\tau$; cf. (3.9).

Case I. We have $\lambda_{*}=(p, q)$ and $\mu_{*}=(p+1, q-1)$. Let $\left(\left(f_{1}, f_{2}\right), \lambda\right)$ be base-data for $z$. Put $y:=e(1, q-1 ; 2, q-1)$. Put $f_{1}(t):=f_{2}$ and, if $q>$ $1, f_{2}(t):=t f_{1}-z f_{2}$. We have

$$
\begin{gathered}
0 \leqslant a \leqslant q-1 \Rightarrow z(t)^{a} f_{1}(t)=z^{a} f_{2}, \\
q \leqslant a \leqslant p \Rightarrow z(t)^{a} f_{1}(t)=t z^{a-1} f_{1}, \\
0 \leqslant a \leqslant q-2 \Rightarrow z(t)^{a} f_{2}(t)=t z^{a} f_{1}-z^{a+1} f_{2}, \\
z(t)^{p+1} f_{1}(t)=0 \text { and } z(t)^{q-1} f_{2}(t)=0 .
\end{gathered}
$$

This implies that $z(t) \in \mathcal{O}(\mu)$ if $t \neq 0$.

Case II. Of the five possibilities, cf. (3.9), we only treat $\left(b_{3}\right)$ and $\left(b_{4}\right)$ with $q \geqslant 2$. The other cases are easier, see [13, (4.3.7)], and already settled in $[10$, pp. 568,569$]$. We choose convenient base-data $\left(\left(f_{1}, \ldots, f_{r}\right), \lambda, \beta, \alpha\right)$ for z. The verifications are left to the reader.

$\left(\mathrm{b}_{3}\right) \lambda_{*}=(p, q, q), p \equiv q \equiv \equiv \in(2), r=3, \beta=\mathrm{id}, \mu_{*}=(p+2, q-1, q$ $-1)$. Choose

$$
\begin{aligned}
y: & y(1, p-1 ; 2,0)+y(1, p-1 ; 3,0) \\
& =e(1,0 ; 2,0)+e(1,0 ; 3,0)+e(2, q-1 ; 1, p-1)-e(3, q-1 ; 1, p-1), \\
f_{1}(t) & :=f_{2}, f_{2}(t):=z f_{2} \text { and } f_{3}(t):=z^{p-q+1} f_{1}-t f_{2}+t f_{3} . \\
& \left(\mathrm{b}_{4}\right) \lambda_{*}=(p, p, q, q), p \equiv q \equiv \epsilon(2), r=4, \beta=\mathrm{id}, \mu_{*}=(p+1, p+1,
\end{aligned}
$$
$q-1, q-1)$. Choose

$$
\begin{aligned}
& y:= y(1, p-1 ; 3,0)+y(1, p-1 ; 4,0) \\
&+y(2, p-1 ; 3,0)+y(2, p-1 ; 4,0) \\
&= e(1,0 ; 3,0)+e(1,0 ; 4,0)+e(2,0 ; 3,0)+e(2,0 ; 4,0) \\
&+e(3, q-1 ; 1, p-1)-e(4, q-1 ; 1, p-1) \\
&-e(3, q-1 ; 2, p-1)+e(4, q-1 ; 2, p-1) \\
& f_{1}(t):=f_{1}, f_{2}(t):=f_{3}, f_{3}(t):=z f_{3} \text { and } f_{4}(t):=z^{p-q+1} f_{1}-t f_{3}+t f_{4} .
\end{aligned}
$$

4. The classical nilpotent scheme, singularities.

(4.1) The symmetrical polynomials $\sigma_{1}, \ldots, \sigma_{n} \in A(\operatorname{End}(F))$ are defined by the equation

$$
\operatorname{det}(x+T \cdot \mathrm{id})=T^{n}+\sum_{m=1}^{n} T^{n-m} \sigma_{m}(x)
$$


in $R[T]$ where $R$ is a $k$-algebra and $x \in \operatorname{End}(F)(R)$. They are invariant under the adjoint action of $G l(F)$ on $\operatorname{End}(F)$. Let $X=\left(x_{i j}\right)$ be the matrix of $x$ with respect to some basis $f_{1}, \ldots, f_{n}$ of $F$. Then

$$
\sigma_{m}(x)=\sum \operatorname{det}\left(x_{i j}\right)_{i, j \in I}
$$

where the summation is over all subsets $I$ of $\{1, \ldots, n\}$ with $\# I=m$.

Case II. Clearly $\sigma_{m} \mid g \in A(g)^{G}$. Let $\Phi$ be the matrix $\phi\left(f_{i}, f_{j}\right)$. We have $x \in g(R)$ if and only if ${ }^{t} X=-\Phi X \Phi^{-1}$. This implies that $\sigma_{m} \mid g=0$ if $m$ is odd. Assume $\epsilon=\zeta=0$. We define $\tau_{l} \in A(g)$ by $\tau_{l}(x):=\operatorname{Pf}(\Phi X)$, where Pf denotes the Pfaffian, cf. $\left[3, \S 5\right.$, no. 2]. Using loc. cit. one proves that $\tau_{l}^{2}=\operatorname{det}(\Phi) \sigma_{n}$ and that $\tau_{l} \in A(\mathrm{~g})^{G}$.

We define the sequence $\mathrm{a}=\left(a_{1}, \ldots, a_{l}\right)$ in $A(g)$ as follows. In Case I we put $a_{i}:=\sigma_{i}$. In Case II $(\epsilon, 1-\epsilon)$ we put $a_{i}:=\sigma_{2 i}$. In Case II $(0,0)$ we put $a_{i}:=\sigma_{2 i}$ if $i<l$, and $a_{l}:=\tau_{l}$.

THEOREM. (a) $A(\mathrm{~g})^{G}$ is the free polynomial ring $k\left[a_{1}, \ldots, a_{l}\right]$.

(b) The sequence a is $A(\mathrm{~g})$-regular (in any order).

(c) $N(G)=\operatorname{Spec}(A(\mathrm{~g}) /\langle\mathrm{a}\rangle)$, it is flat over $k$.

(d) $N(G)$ is smooth over $k$ in the points of $D(v)$ where $v$ is, cf. (3.8)(b).

(e) If $k$ is a normal ring then $N(G)$ is a normal scheme.

Proof. (a) Let $u: k\left[T_{1}, \ldots, T_{l}\right] \rightarrow A(g)^{G}$ be defined by $T_{i} \mapsto a_{i}$. We have to prove that $u$ is bijective. Replacing $k$ by a faithfully flat $k$-algebra (cf. (3.6) and (3.2)), we may assume the existence of a standard nilpotent $z \in g(k)$ with partition $\nu, \mathrm{cf}$. (3.8)(b). Let $z+L$ be the cross section of (3.7). By (2.2) the morphism $v: A(g) G \rightarrow A(z+L)$ is injective. Case by case one shows that $v \circ u$ is bijective, so that $u$ is bijective.

(b) and (c). By [7], Theorem (2.6) applies. So $A(g)$ is flat over $A(g)^{G}$. So we have (b) and (c).

(d) We may use the cross section of (a). Now $(z+L) \cap N(G)$ is a cross section at $z$ for the action of $G$ on $N(G)$, and the assertion follows from $(z+L)$ $\cap N(G) \cong \operatorname{Spec}(k)$.

(e) By (c) and [12, IV 6.14.1] we may assume that $k$ is a field. Now $N(G)$ is nonsingular in codimension one, by (d) and (3.8)(b). So $N(G)$ is normal by Serre's criterion [12, IV 5.8.6].

REMARKS. (i) There are other ways to prove the theorem, either avoiding (3.7) or avoiding (2.6) and [7]. (ii) It can be shown that $N(S l(F)$ ) exists and is equal to $N(G l(F))$, where $k$ is arbitrary. Here (2.6) does not apply.

(4.2) If $\lambda \in P(n)$, the partition $\Sigma \lambda$ is defined in (3.9). Case II $(\epsilon, 1-\epsilon)$. If $\lambda \in P_{\epsilon}(n)$ where $n=2 l+1-\epsilon$, then we define the partition $\Sigma_{\epsilon} \lambda$ by $\left(\Sigma_{\epsilon} \lambda\right)_{i}$ $:=(\Sigma \lambda)_{2 i-\epsilon}$. Case II $(0,0)$. If $\lambda \in P_{0}(n)$ where $n=2 l$, then we define $\Sigma_{0} \lambda$ 
$:=\theta+\nu$ where $\theta, \nu \in P$ are given by $\theta_{i}:=(\Sigma \lambda)_{2 i+1}$ and $\nu_{*}:=\left(1 / 2 \lambda^{1}-1\right)$. Note: in the last case $\lambda^{1}$ is even and $(\Sigma \lambda)_{1}=\lambda^{1}-1$. We write $\Sigma_{(\epsilon)}$ to denote $\Sigma$ in Case I and $\Sigma_{\epsilon}$ in Case II. $\Sigma_{(1)}$ means that $\epsilon=0$ is excluded in Case II.

Theorem. Consider $x \in \mathfrak{D}(\lambda)$. Then ord $(x, N(G) / k)=\Sigma_{(1)} \lambda$ in Cases I and II $(1,0)$, and ord $(x, N(G) / k) \geqslant \Sigma_{0} \lambda$ in Case II $(0, \zeta)$.

Proof. (1) By (1.7) we may replace $(N(G), x)$ by a smoothly equivalent pointed scheme. So by (3.6) and (3.2) we may assume the existence of a standard nilpotent $z \in \mathfrak{g}(k)$ with partition $\lambda$. By (2.1) (a) we may assume that $x=z(\mathfrak{p})$ for some $\mathfrak{p} \in \operatorname{Spec}(k)$. Put $A:=\mathcal{O}_{\beta, x}$ and $B:=\mathcal{O}_{N(G), x}$. We have $B=A /\langle\mathrm{a}\rangle$ where a is the $A$-regular sequence of (4.1), or rather its image in $A$.

(2) Let $J$ be the ideal in $A(g)$ corresponding to the section $z$. So $x$ corresponds to the prime ideal $J+p A(g)$. We claim

(a) If $1 \leqslant i \leqslant n$ and $m:=1+(\Sigma \lambda)_{n+1-i}$ then $\sigma_{i} \in J^{m}$.

(b) In Case II $(0,0)$ we have $\tau_{l} \in J^{m}$ where $m:=1 / 2 \lambda^{1}$.

Proof of (a). It suffices to consider Case I. Let (f, $\lambda$ ) be base-data for $z$. Using the notation of (3.7) we define

$$
\sigma_{P}:=\operatorname{det} \xi\left(\psi ; \psi^{\prime}\right)_{\psi, \psi^{\prime} \in P}
$$

if $\varnothing \neq P \subset \Psi$. So $\sigma_{i}=\Sigma \sigma_{P}$ where the summation is over all $P$ with $\# P=i$. If $\xi\left(\psi ; \psi^{\prime}\right) \notin J$ then we have $\psi^{\prime}=(j, a), \psi=(j, a+1)$ for some $j$ and $a$. Consider $P$ with $\# P=i$. If $\pi$ is a permutation of $P$ then one verifies that

$$
\#\{(j, a) \in P \mid \pi(j, a) \neq(j, a+1)\} \geqslant 1+(\Sigma \lambda)_{n+1-i}=m .
$$

This implies $\sigma_{P} \in J^{m}$, proving (a).

PROOF OF (b). We may assume that $k$ is reduced. Now the assertion follows from $\tau_{l}^{2}=\operatorname{det}(\Phi) \sigma_{n} \in J^{2 m}$, cf. (a).

(3) By (1.6)(a) it follows from (2)(a), (b) that ord $(B / k) \geqslant \Sigma_{(\epsilon)} \lambda$. This proves the theorem in Case II $(0, \zeta)$. In the rest of the proof Case II $(0, \zeta)$ is excluded. It suffices to prove

$$
\operatorname{ord}(B / k) \leqslant \Sigma_{(1)} \lambda \text {. }
$$

By (1.5) and (4.1)(c) we may replace $k$ by an algebraic closure of the field $\mathrm{k}(\mathfrak{p})$. So henceforth $k$ is an algebraically closed field. Now $x$ and $z$ may be identified. Let $(f, \lambda)$, resp. ( $f, \lambda, \beta, \alpha)$ be its base-data.

(4) We prove (3) (*) by induction on $n=|\lambda|$. The cases with $n \leqslant 1$ are trivial. So assume $n \geqslant 2$. Put $r:=\lambda^{1}$. Let $\rho$ be the partition with $\rho_{*}=(r-1)$. The partition $\mu$ is defined as follows.

Case I. $\mu_{r}:=\lambda_{r}-1, \mu_{i}:=\lambda_{i}$ if $i \neq r$.

Case II. If $\lambda_{r}$ is even then $\mu_{r}:=\lambda_{r}-2$ and $\mu_{i}:=\lambda_{i}$ otherwise. If $\lambda_{r}$ is 
odd so that $\lambda_{r-1}=\lambda_{r}$, then $\mu_{r-1}:=\mu_{r}:=\lambda_{r}-1$ and $\mu_{i}:=\lambda_{i}$ otherwise.

One verifies that $\mu \in P_{(1)}$ and that $\Sigma_{(1)} \lambda=\rho+\Sigma_{(1)} \mu$.

We have $F=\Sigma k f(\psi), \psi \in \Psi$, cf. (3.7). Let $P$ be the subset of $\Psi$ containing $(r, 0)$ and in Case II also $\left(\beta r, \lambda_{r}-1\right)$. Put $F^{\prime}:=\Sigma k f(\psi), \psi \notin P$, and $F^{\prime \prime}:=$ $\Sigma k f(\psi), \psi \in P$. Clearly $F=F^{\prime} \oplus F^{\prime \prime}$. In Case II the form $\phi^{\prime}:=\phi \mid F^{\prime}$ is nondegenerate and hence a 1 -form on $F^{\prime}$. We put $G^{\prime}:=G l\left(F^{\prime}\right)$ in Case I and $G^{\prime}:=$ $G\left(F^{\prime}, \phi^{\prime}\right)$ in Case II. So the convention (3.1) concerning $G^{\prime}$ is not applied here. We put $\mathfrak{g}^{\prime}:=\operatorname{Lie}\left(G^{\prime}\right)$, etc.

Let

$$
\left(\begin{array}{ll}
x^{\prime} & x_{2} \\
x_{1} & x_{3}
\end{array}\right)
$$

be the matrix of $x$ with respect to the decomposition $F=F^{\prime} \oplus F^{\prime \prime}$. Now $x^{\prime}$ is a standard nilpotent in $\mathfrak{g}^{\prime}(k)$ with partition $\mu$. Consider the ring

$$
B^{\prime}:=\mathcal{O}_{N\left(G^{\prime}\right), x^{\prime}}=\mathcal{O}_{g^{\prime}, x^{\prime}} /\left\langle\left(\sigma_{i}^{\prime}\right)_{i<n^{\prime}}\right\rangle .
$$

By induction we have ord $\left(B^{\prime} / k\right) \leqslant \Sigma_{(1)} \mu$. One verifies that

$$
w \mapsto\left(\begin{array}{ll}
w & x_{2} \\
x_{1} & x_{3}
\end{array}\right)
$$

defines a regular immersion $u: g^{\prime} \rightarrow g$ such that $u\left(x^{\prime}\right)=x, u^{0}\left(\sigma_{n}\right)=0$ and $u^{0}\left(\sigma_{i}\right)=\sigma_{i}^{\prime}$ if $i<n$, where $u^{0}: A(g) \rightarrow A\left(g^{\prime}\right)$ is the comorphism. Put $R:=$ $A /\left\langle\left(\sigma_{i}\right)_{i<n}\right\rangle$ so that $B=R /\langle f\rangle$ where $f$ is the image of $\sigma_{n}$ in $R$. Now there is an $R$-regular sequence $\mathrm{x}$ in $R$ such that $B^{\prime} \cong R /\langle\mathrm{x}\rangle$ and $f \in\langle\mathrm{x}\rangle$. By $(1.6)(\mathrm{b})$ this implies

$$
\operatorname{ord}(B / k) \leqslant \rho+\operatorname{ord}\left(B^{\prime} / k\right) \leqslant \rho+\Sigma_{(1)} \mu=\Sigma_{(1)} \lambda
$$

provided that $f \notin m_{R}^{r+1}$. So in order to prove the theorem it suffices to prove that

$$
\sigma_{\Psi} \notin\left\langle\left(\sigma_{P}\right)_{P \neq \Psi}\right\rangle+m_{A}^{r+1}
$$

where we have used the notation of (2).

(5) In Case II we normalize the base-data of $x$ as follows: $\beta i \neq i$ if and only if $\lambda_{i}$ is odd; $|\beta i-i| \leqslant 1$ for all $i$; if $i \geqslant \beta i$ then $\alpha(i)=1$. Now $i \leqslant \beta i$ implies $\alpha(i)=(-1)^{\lambda_{i}}$. With the notation of (3.7) we define a linear subvariety $M$ of 8 .

Case I. $M:=\Sigma k e\left(i, 0 ; j, \lambda_{j}-1\right)(1 \leqslant i, j \leqslant r)$.

Case II. $M:=\Sigma k y\left(i, \lambda_{i}-1 ; j, \lambda_{j}-1\right)$ where the summation is over all pairs $(i, j)$ such that $i=j$ or $i \leqslant \beta i<j \leqslant \beta j$. So in this case $M \subset M_{I}$.

The ring $A(x+M)$ is considered as a graded $k$-algebra such that $x$ corresponds to the augmentation ideal. The functions $\sigma_{p} \mid x+M$ are homogeneous, 
$\sigma_{\Psi} \mid x+M$ is homogeneous of degree $r$. So it suffices to prove

$$
\sigma_{\Psi} \mid x+M \notin\left\langle\left(\sigma_{P} \mid x+M\right)_{P \neq \Psi}\right\rangle .
$$

(6) Case I. It is easy to see that $x+M$ has a subvariety $x_{1}+M_{1}$ such that $\sigma_{P} \mid x_{1}+M_{1} \neq 0$ if and only if $P=\Psi$. This proves $(5)(*)$ and the theorem.

Case II. Consider the subvariety $x_{1}+M_{1}$ of $x+M$ where

$$
\begin{aligned}
& x_{1}:=x+\sum_{i>\beta i} y\left(i, \lambda_{i}-1 ; i, \lambda_{i}-1\right), \\
& M_{1}:=\sum k y\left(i, \lambda_{i}-1 ; j, \lambda_{j}-1\right)(i \leqslant \beta i, i \leqslant j \leqslant \beta j) .
\end{aligned}
$$

Now $x_{1}$ is a standard nilpotent in $g_{I}(k)$ with base-data $\left(f^{\prime}, \lambda^{\prime}\right)$ such that

$$
M_{1}=\sum_{i<j} k\left(e^{\prime}\left(i, 0 ; j, \lambda_{j}^{\prime}-1\right)+e^{\prime}\left(j, 0 ; i, \lambda_{i}^{\prime}-1\right)\right)
$$

with respect to the new base-data. In order to prove $(5)(*)$ and hence the theorem, it suffices to show that

$$
\sigma_{\Psi} \mid x_{1}+M_{1} \notin\left\langle\left(\sigma_{P} \mid x_{1}+M_{1}\right)_{P \neq \Psi}\right\rangle .
$$

This is a consequence of the following:

LEMmA. Assume char $(k) \neq 2$. Let $r \in \mathrm{N}$. Consider the ring $k\left[T_{i j}\right]$ where $1 \leqslant i \leqslant j \leqslant r$. Put $T_{i j}:=T_{j i}$ if $i>j$. Put $Q:=\{1, \ldots, r\}$. If $\varnothing \neq P \subset Q$

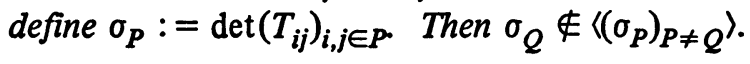

Proof. We may assume $r \geqslant 3$. Let $I$ be the ideal generated by all $T_{i j}$ such that $1 \neq|i-j| \neq r-1$, and all $T_{i j}^{2}$. It is easy to see that $\sigma_{P} \notin I$ if and only if $P=Q$.

(4.3) The following facts are not proved here, see [13, pp. 11-13].

(i) The mapping $\Sigma_{(1)}: P_{(1)}(n) \rightarrow P$ is injective.

(ii) If $\Sigma_{1} \lambda \leqslant \Sigma_{1} \mu$ where $\lambda, \mu \in P_{1}(n)$ then $\Sigma \lambda \leqslant \Sigma \mu$.

(iii) If $\lambda \in P_{(1)}(n)$ then $\gamma_{(1)}(\lambda)=2\left|\Sigma_{(1)} \lambda\right|$.

Using (1.7), (2.1)(a), (3.5), (3.8), (3.10), (4.2) we get the following

Corollary. Case I and II $(1,0)$. Let $x \in \mathfrak{D}(\lambda)$ and $y \in N(G)$.

(a) $y \in \mathfrak{D}(\lambda)$ if and only if $\operatorname{ord}(y, N(G) / k)=\operatorname{ord}(x, N(G) / k)$.

(b) $y \in G x$ if and only if $(N(G), y) \sim(N(G), x), c f$. (1.7).

Assume that $k$ is a field.

(c) $\operatorname{codim}(G x, N(G))=2 \operatorname{lord}(x, N(G) / k) \mid$.

(d) $y \in \overline{G x}$ if and only if $\operatorname{ord}(y, N(G) / k) \geqslant \operatorname{ord}(x, N(G) / k)$.

(4.4) REMARK. In (4.2) Case II $(0, \zeta)$, inequality occurs if $\lambda_{1}$ is even and also if $\lambda_{*}=(3,3,2,2)$, but we have equality if $\lambda_{*}=(3,3,2,2,1)$. In the last case we have 


$$
\operatorname{codim}(G x, N(G))=\gamma_{0}(\lambda)>2\left|\Sigma_{0} \lambda\right|=2|\operatorname{ord}(x, N(G) / k)|
$$

if $k$ is a field, compare (4.3)(c) and (4.9) table $B_{5}$.

(4.5) The polynomials $f_{a}$ are defined by $f_{a}:=0$ if $a<0, f_{0}:=1$ and $f_{a}:=\Sigma_{i \geqslant 1} X_{i} f_{a-i}$ if $a>0$. They are determined by the generating function

$$
\sum_{a=0}^{\infty} T^{a} f_{a}=\left(1-\sum_{i \geqslant 1} X_{i} T^{i}\right)^{-1}
$$

Clearly $f_{a}\left(X_{1}\right)=X_{1}^{a}$ if $a \geqslant 0$. One can prove that

$$
f_{a}\left(X_{1}, X_{2}\right)=\sum\left(\begin{array}{c}
a-i \\
i
\end{array}\right) X_{1}^{a-2 i} X_{2}^{i} \quad(0 \leqslant i \leqslant 1 / 2 a) .
$$

Let $\mathbf{A}^{m}$ denote the affine space over $k$ of rank $m$, say with coordinate ring $k\left[X_{1}, \ldots, X_{m}\right]$. It is pointed in some point of the origin section. The Kleinian singularities $A_{l}$ and $D_{l}$ are the pointed subschemes of $\mathrm{A}^{3}$ given by one equation:

$A_{l}, l \geqslant 1$, by $X_{1}^{l+1}+X_{2} X_{3}=0$,

$D_{l}, l \geqslant 3$, by $X_{1}^{l-1}-X_{1} X_{2}^{2}+X_{3}^{2}=0$, if $1 / 2 \in k$.

We define the following singularities.

If $l \geqslant 3, A A_{1}$ in $\mathrm{A}^{2} \times \mathrm{A}^{4}$ by

$$
\left\{\begin{array}{l}
f_{l}\left(X_{1}, X_{2}\right)+Y_{1} Y_{3}+Y_{2} Y_{4}=0 \\
X_{2} f_{l-1}\left(X_{1}, X_{2}\right)-Y_{4}\left(X_{1} Y_{2}-X_{2} Y_{1}\right)+Y_{2} Y_{3}=0 .
\end{array}\right.
$$

If $1 / 2 \in k$ and $l \geqslant 3, B B_{l}$ in $\mathbf{A}^{2} \times \mathbf{A}^{4}$ by

$$
\left\{\begin{array}{l}
f_{l-1}\left(2 X_{1},-X_{2}^{2}\right)-2 Y_{1} Y_{3}+Y_{2}^{2}-Y_{4}^{2}=0, \\
X_{2}^{2} f_{l-2}\left(2 X_{1},-X_{2}^{2}\right)+\left(Y_{3}-X_{1} Y_{1}\right)^{2}-X_{2}^{2} Y_{1}^{2}-2 Y_{4}\left(X_{1} Y_{4}-X_{2} Y_{2}\right)=0 .
\end{array}\right.
$$

If $1 / 2 \in k$ and $l \geqslant 2, C C_{l}$ in $\mathrm{A}^{3} \times \mathrm{A}^{2}$ by

$$
\left(X_{3}^{2}-X_{1} X_{2}\right)^{l}+X_{1} Y_{1}^{2}+2 X_{3} Y_{1} Y_{2}+X_{2} Y_{2}^{2}=0 \text {. }
$$

If $1 / 2 \in k$ and $l \geqslant 5, C D_{l}$ in $\mathrm{A}^{2} \times \mathrm{A}^{4}$ by

$$
\left\{\begin{array}{l}
f_{l-2}\left(X_{1}, X_{2}\right)+X_{1} Y_{2}^{2}-X_{2} Y_{1}^{2}-Y_{3}^{2}+2 Y_{2} Y_{4}=0 \\
X_{2} f_{l-3}\left(X_{1}, X_{2}\right)+X_{2}\left(X_{1} Y_{1}^{2}+Y_{2}^{2}-2 Y_{1} Y_{3}\right)+Y_{4}^{2}=0 .
\end{array}\right.
$$

If $1 / 2 \in k$ and $l \geqslant 3, D D_{l}$ in $\mathrm{A}^{3} \times \mathrm{A}^{3}$ by

$$
\left\{\begin{array}{l}
\left(x_{1}^{2}+x_{2}^{2}+x_{3}^{2}\right)^{l-1}+y_{1}^{2}+y_{2}^{2}+y_{3}^{2}=0 \\
x_{1} y_{1}+x_{2} y_{2}+x_{3} y_{3}=0
\end{array}\right.
$$


(4.6) Proposition. Assume in Case II that $l+\epsilon+\zeta \geqslant 3$. Consider $\lambda \epsilon$ $P_{(\epsilon)}(n)$ with $0<\gamma_{(\epsilon)}(\lambda)<6, c f$. (3.8). If $x \in \mathcal{D}(\lambda)$ then $(N(G), x)$ is smoothly equivalent (cf. (1.7)) to the singularity (cf. (4.5)) given in the following table.

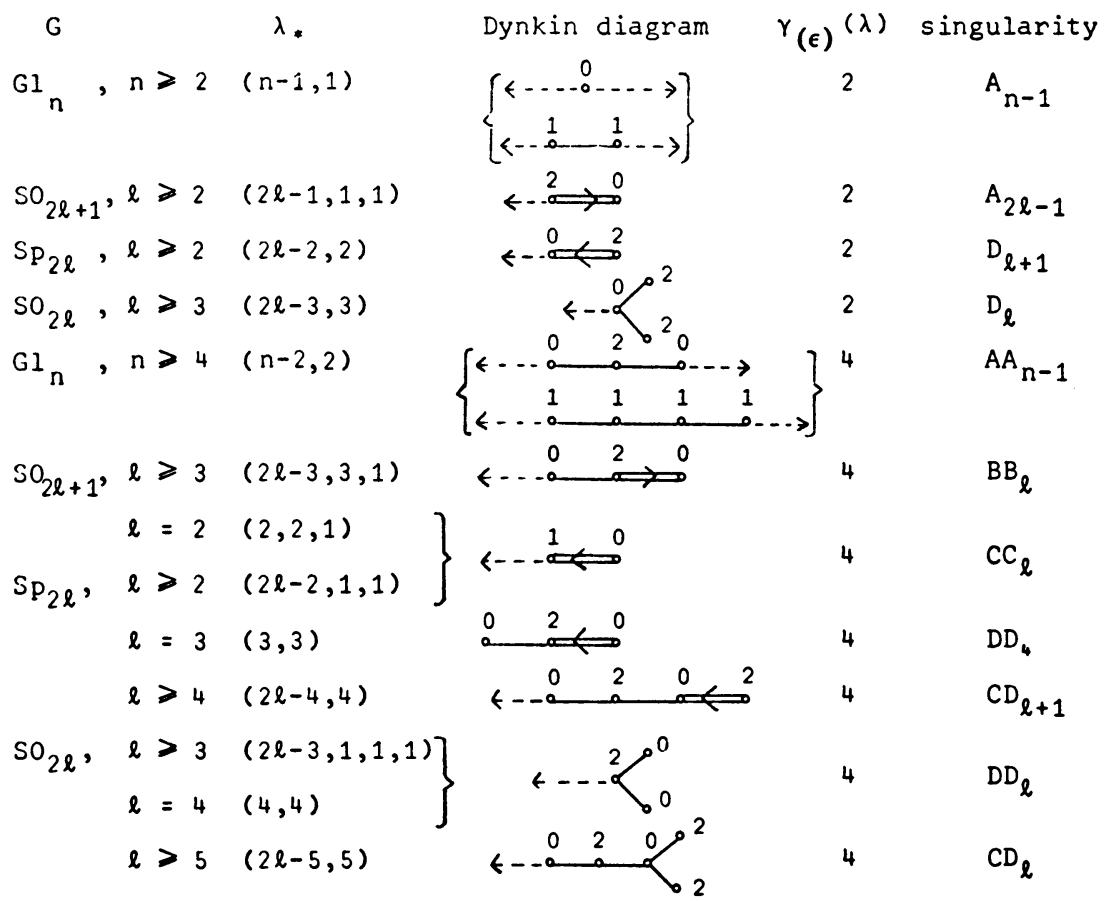

REMARK. We have $\gamma_{(\epsilon)}(\lambda)=\operatorname{codim}\left(G x, N(G)_{(\mathrm{k}(x))}\right)$. For the singularities with $\gamma_{(\epsilon)}(\lambda)=2$, compare [5] and [21, pp. 140-158]. In the table we have added the Dynkin diagram of the section $x \in g(k(x))$, cf. [20, III, IV], where $\leftarrow--$ means a string with numbers 2 attached to the nodes.

Proof. The classification of all possibilities for $\lambda$ is easy. By the sequence of reductions used in (4.2)(1) we may assume that $x=z(\mathfrak{p})$ where $z$ is a standard nilpotent with partition $\lambda$ and $p \in \operatorname{Spec}(k)$. In Case II the base-data for $z$ may be prescribed within the bounds set by (3.3)(2). Let $z+L$ be the cross section of (3.7). Then $(z+L) \cap N(G)$ is a cross section at $z$ for the action of $G$ on $N(G)$. So $(N(G), x)$ is smoothly equivalent to $((z+L) \cap N(G), z(p))$ by $(2.1)$ (a). The two singularities to be determined for $G l_{n}$ will be examples in (4.7) and (4.8). We do not give the tedious calculations needed to settle Case II, see $[13$, p. 79$]$ for some indications.

(4.7) Case I with $\lambda_{*}=\left(p, 1^{q}\right)$, i.e. $(p, 1, \ldots, 1)$ with $q$ times 1 . We have $n=p+q$ and $r:=\lambda^{1}=q+1$. On $z+L$ we define the coordinate functions $\xi_{a}, \xi_{i j}$ as follows: if $R$ is a $k$-algebra and $x \in(z+L)(R)$, then 
(1) $x=z-\sum_{a=1}^{p} \xi_{a}(x) e(1, p-a ; 1, p-1)-\sum_{(i, j) \neq(1,1)} \xi_{i j}(x) e\left(i, 0 ; j, \lambda_{j}-1\right)$.

So $A(z+L)=k\left[\xi_{a}, \xi_{j i}\right]$. Put $\xi_{11}=0$.

If $a \geqslant 1$, let $s_{a}, h_{a} \in k\left[\xi_{i j}\right]$ be defined by

$$
\left\{\begin{array}{l}
s_{a}=\sum \operatorname{det}\left(\xi_{i j}\right)_{i, j \in I} \\
h_{a}=\sum \operatorname{det}\left(\xi_{i j}\right)_{i, j \in\{1\} \cup I}
\end{array}\right.
$$

where in both cases the summation is over the subsets $I$ of $\{2, \ldots, r\}$ with $\# I=a$.

Clearly, if $a \geqslant r$ then $s_{a}=h_{a}=0$. The subscheme $(z+L) \cap N(G)$ of $z+L$ is defined by the equations $\sigma_{m} \mid(z+L)=0(1 \leqslant m \leqslant n)$. One verifies that

$$
\begin{cases}(-1)^{m} \sigma_{m} \mid(z+L)=\xi_{m}+s_{m}+\sum_{a=1}^{m-1} \xi_{a} s_{m-a} & \text { if } 1 \leqslant m \leqslant p, \\ (-1)^{m} \sigma_{m} \mid(z+L)=h_{m-p}+s_{m}+\sum_{a=1}^{p} \xi_{a} s_{m-a} & \text { if } p<m \leqslant n .\end{cases}
$$

The first $p$ equations can be solved inductively. With the notations of (4.5) we obtain $\xi_{m}=f_{m}\left(-s_{1},-s_{2}, \ldots, s_{q}\right)(1 \leqslant m \leqslant p)$. So $(z+L) \cap N(G)$ is isomorphic to the subscheme of Spec $k\left[\xi_{i j}\right]$ defined by the equations

$$
h_{m-p}+\sum_{a=0}^{p} s_{m-a} f_{a}\left(-s_{1}, \ldots,-s_{q}\right)=0 \quad(p<m \leqslant n) .
$$

EXAMPLES. (a) $\lambda_{*}=(n-1,1)$. Putting $X_{1}=-\xi_{22}, X_{2}=\xi_{12}, X_{3}=\xi_{21}$, we get the singularity $A_{n-1}$, cf. (4.5).

(b) $\lambda_{*}=(n-2,1,1)$. The scheme $(z+L) \cap N(G)$ is isomorphic to the subscheme of $A^{8}=\operatorname{Spec}\left(k\left[\xi_{i j}\right]\right)$, where $1 \leqslant i, j \leqslant 3 \leqslant i+j$, defined by the equations

$$
\left\{\begin{array}{l}
f_{n-1}\left(-s_{1},-s_{2}\right)-h_{1}=0, \\
s_{2} f_{n-2}\left(-s_{1},-s_{2}\right)+h_{2}=0,
\end{array}\right.
$$

where

$$
\begin{aligned}
& s_{1}=\xi_{22}+\xi_{33}, \\
& s_{2}=\xi_{22} \xi_{33}-\xi_{23} \xi_{32}, \\
& h_{1}=\xi_{12} \xi_{21}+\xi_{13} \xi_{31},
\end{aligned} \quad \text { and } \quad h_{2}=\left|\begin{array}{lll}
0 & \xi_{12} & \xi_{13} \\
\xi_{21} & \xi_{22} & \xi_{23} \\
\xi_{31} & \xi_{32} & \xi_{33}
\end{array}\right| .
$$

(4.8) Case I for arbitrary $\lambda$. We use a different cross section, viz. $z+L^{\prime \prime}$ defined by $L^{\prime \prime}:=\Sigma_{i, j} L_{i j}^{\prime \prime}$ where $L_{i j}^{\prime \prime}:=L_{i j}$ if $i \neq 1$ or $j=1$ and $L_{1, j}^{\prime \prime}(R):=$ $\Sigma_{0<b<\lambda_{j}} \operatorname{Re}(1,0 ; j, b)$ if $j \neq 1$, see (3.7). Again we have 


$$
(N(G), x) \sim\left(\left(z+L^{\prime \prime}\right) \cap N(G), z(\mathfrak{p})\right) .
$$

Put $p:=\lambda_{1}, q:=n-\lambda_{1}$ and $\mu_{*}:=\left(p, 1^{q}\right)$. Put $z^{\prime}:=\Sigma_{a=0}^{p-2} e(1, a+1 ; 1, a)$, so that $z^{\prime}$ is a standard nilpotent element in $g(k)$ with partition $\mu$. In the obvious way we define base-data $\left(f^{\prime}, \mu\right)$ for $z^{\prime}$. The cross section $z^{\prime}+L^{\prime}$ at $z^{\prime}$ used in (4.7) contains $z+L^{\prime \prime}$. So we can use the elimination in (4.7) of $\xi_{a}, 1 \leqslant a \leqslant p$, substituting into the matrix $\left(\xi_{i j}\right)$ at some places the constant functions 0 or $-1, \mathrm{cf}$. (4.7)(1).

EXAMPLE. If $\lambda_{*}=(n-2,2), n \geqslant 4$, we use the matrix

$$
\left(\xi_{i j}\right)=\left(\begin{array}{ccc}
0 & Y_{3} & Y_{4} \\
Y_{1} & -X_{1} & -1 \\
Y_{2} & -X_{2} & 0
\end{array}\right)
$$

and we obtain the equations (4.7)(5) where $s_{1}=-X_{1}, s_{2}=-X_{2}, h_{1}=Y_{1} Y_{3}+$ $Y_{2} Y_{4}$ and $h_{2}=Y_{4}\left(X_{1} Y_{2}-X_{2} Y_{1}\right)-Y_{2} Y_{3}$. So $\left(z+L^{\prime \prime}\right) \cap N(G)$ is isomorphic to the singularity $A A_{n-1}$, cf. (4.5).

(4.9) Tables for the orbits in $N(G)$. We give the adjacency structure (cf. (3.10)), the Dynkin diagram (cf. [20, IV]), the codimension of the orbits $\gamma_{(\epsilon)}(\lambda)$ (cf. (3.8)), and the partition ord $=\operatorname{ord}(x, N(G) / k)$ (cf. (4.2)). The number of orbits is denoted by \#. In the cases $S_{2 l}$ with even $l$, the partition $\lambda$ may represent two orbits, cf. (3.5). We give the Dynkin diagram of one of them and indicate how to get the other one by the symbol $\curvearrowleft$.

For $S O_{n}$ we give $\Sigma_{0} \lambda$, which is a lower bound of ord, cf. (4.2). Whenever there are reasons to assume ord $\neq \Sigma_{0} \lambda$, we give a conjectured value of ord or a question mark. As $D_{2}=A_{1}+A_{1}, B_{2}=C_{2}$ and $D_{3}=A_{3}$, the values of ord for the cases $\mathrm{SO}_{4}, \mathrm{SO}_{5}$ and $\mathrm{SO}_{6}$ are not conjectural.

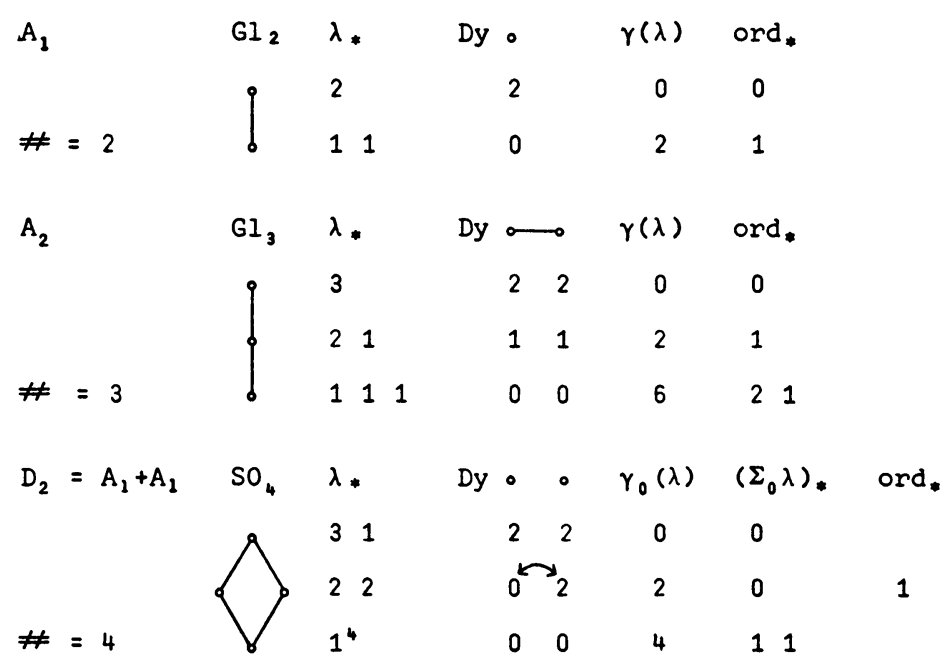




\begin{tabular}{|c|c|c|c|c|c|c|c|}
\hline$B_{2}=C_{2}$ & $\mathrm{SO}_{5}$ & $\lambda$ * & Dy & & $\gamma_{0}(\lambda)$ & $\left(\Sigma_{0} \lambda\right)$. & ord* \\
\hline & 9 & 5 & 2 & 2 & 0 & 0 & 0 \\
\hline & & $\begin{array}{lll}3 & 1 & 1\end{array}$ & 2 & 0 & 2 & 1 & .1 \\
\hline & & 221 & 0 & 1 & 4 & 1 & 2 \\
\hline$=4$ & ! & $1^{5}$ & 0 & 0 & 8 & $\begin{array}{ll}31 \\
\text { I }\end{array}$ & \\
\hline
\end{tabular}

$C_{2}=B_{2} \quad S_{4} \quad \lambda * \quad$ Dy $\Longleftrightarrow r_{1}(\lambda)$ ord.

$\#=4 \quad 1 \begin{array}{llllll} & 14 & 0 & 0 & 8 & 2\end{array}$

\begin{tabular}{|c|c|c|c|c|c|c|c|}
\hline \multirow{5}{*}{$A_{3}=D_{3}$} & GI. & $\lambda$ & \multicolumn{3}{|c|}{ 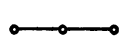 } & $\gamma(\lambda)$ & ord. \\
\hline & 9 & 4 & 2 & 2 & 2 & 0 & 0 \\
\hline & & 31 & 2 & 0 & 2 & 2 & 1 \\
\hline & & 22 & 0 & 2 & 0 & 4 & 11 \\
\hline & & $\begin{array}{lll}2 & 1 & 1\end{array}$ & 1 & 0 & 1 & 6 & 21 \\
\hline$\#=5$ & ! & $1^{4}$ & 0 & 0 & 0 & 12 & $\begin{array}{lll}32 & 1\end{array}$ \\
\hline
\end{tabular}

\begin{tabular}{|c|c|c|c|c|c|c|c|c|}
\hline \multirow[t]{5}{*}{$D_{3}=A_{3}$} & so, & $\lambda$ * & $\propto$ & & & $\gamma_{0}(\lambda)$ & $\left(\Sigma_{0} \lambda\right)$ & ord* \\
\hline & $p$ & 51 & 2 & 2 & 2 & 0 & 0 & \\
\hline & & 33 & 0 & 2 & 2 & 2 & 1 & \\
\hline & & $\begin{array}{llll}3 & 1 & 1 & 1\end{array}$ & 2 & 0 & 0 & 4 & 11 & \\
\hline & & 2211 & 0 & 1 & 1 & 6 & 11 & 21 \\
\hline$\neq=5$ & 1 & $1^{6}$ & 0 & 0 & 0 & 12 & $\begin{array}{lll}32 & 1\end{array}$ & \\
\hline
\end{tabular}

\begin{tabular}{|c|c|c|c|c|c|c|c|c|}
\hline$B_{3}$ & SO, & $\lambda$ & $a$ & $\Leftrightarrow$ & & $\gamma_{0}(\lambda)$ & $\left(\Sigma_{0} \lambda\right)_{*}$ & ord. \\
\hline & $i$ & 7 & 2 & 2 & 2 & 0 & 0 & \\
\hline & & $\begin{array}{lll}5 & 1 & 1\end{array}$ & 2 & 2 & 0 & 2 & 1 & \\
\hline & & $\begin{array}{lll}3 & 3 & 1\end{array}$ & 0 & 2 & 0 & 4 & 11 & \\
\hline & & 322 & 1 & 0 & 1 & 6 & 21 & \\
\hline & & $31^{4}$ & 2 & 0 & 0 & 8 & 31 & \\
\hline & & $221^{3}$ & 0 & 1 & 0 & 10 & 31 & 32 \\
\hline$\#=7$ & 0 & $1^{7}$ & 0 & 0 & 0 & 18 & $\begin{array}{lll}5 & 3 & 1\end{array}$ & \\
\hline
\end{tabular}




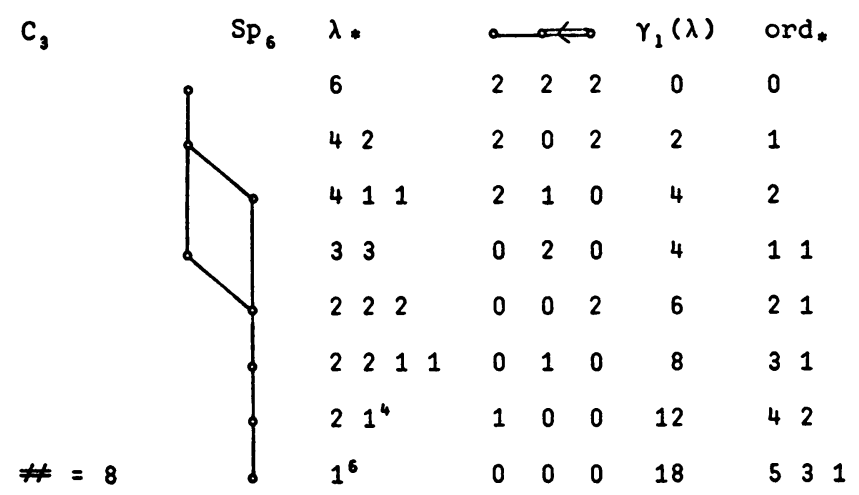

\begin{tabular}{|c|c|c|c|c|c|c|c|c|}
\hline \multirow[t]{7}{*}{$\mathrm{A}_{4}$} & \multirow{2}{*}{$\begin{array}{c}G I_{5} \\
q\end{array}$} & \multirow[t]{2}{*}{$\lambda_{*}$} & \multicolumn{4}{|c|}{ م } & $\gamma(\lambda)$ & ora. \\
\hline & & & 2 & 2 & 2 & 2 & 0 & 0 \\
\hline & & 41 & 2 & 1 & 1 & 2 & 2 & 1 \\
\hline & & 32 & 1 & 1 & 1 & 1 & 4 & 11 \\
\hline & & $\begin{array}{lll}3 & 1 & 1\end{array}$ & 2 & 0 & 0 & 2 & 6 & 21 \\
\hline & & 221 & 0 & 1 & 1 & 0 & 8 & 211 \\
\hline & & $21^{3}$ & 1 & 0 & 0 & 1 & 12 & $\begin{array}{lll}32 & 2\end{array}$ \\
\hline$\#=7$ & & $1^{5}$ & 0 & 0 & 0 & 0 & 20 & 432 \\
\hline
\end{tabular}

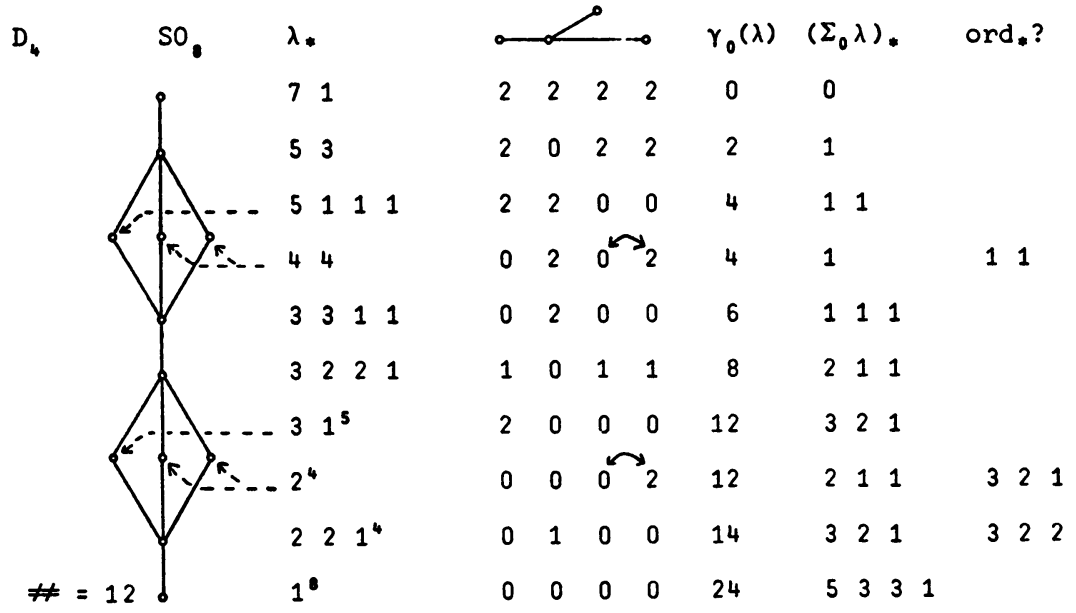




\begin{tabular}{|c|c|c|c|c|c|c|c|c|c|c|c|}
\hline \multirow[t]{13}{*}{$B_{4}$} & so, & $\lambda$ * & \multicolumn{4}{|c|}{$\circ \longrightarrow 0$} & $\gamma_{0}(\lambda)$ & \multicolumn{2}{|c|}{$\left(\Sigma_{0} \lambda\right)$} & \multicolumn{2}{|c|}{ ord *? } \\
\hline & & 9 & 2 & 2 & 2 & 2 & 0 & 0 & & & \\
\hline & & $\begin{array}{lll}7 & 1 & 1\end{array}$ & 2 & 2 & 2 & 0 & 2 & 1 & & & \\
\hline & & $\begin{array}{lll}5 & 3 & 1\end{array}$ & 2 & 0 & 2 & 0 & 4 & 1 & 1 & & \\
\hline & & 522 & 2 & 1 & 0 & 1 & 6 & 2 & 1 & & \\
\hline & & $51^{4}$ & 2 & 2 & 0 & 0 & 8 & 3 & 1 & & \\
\hline & & 441 & 0 & 2 & 0 & 1 & 6 & 1 & 1 & 2 & 1 \\
\hline & & $\begin{array}{lll}3 & 3 & 3\end{array}$ & 0 & 0 & 2 & 0 & 8 & 2 & 11 & & \\
\hline & & $331^{3}$ & 0 & 2 & 0 & 0 & 10 & 3 & 11 & & \\
\hline & & $\begin{array}{lllll}3 & 2 & 2 & 1 & 1\end{array}$ & 1 & 0 & 1 & 0 & 12 & 3 & 21 & & \\
\hline & & $31^{6}$ & 2 & 0 & 0 & 0 & 18 & 5 & 31 & & \\
\hline & & $2^{4} 1$ & 0 & 0 & 0 & 1 & 16 & 3 & 21 & 3 & 32 \\
\hline & & $221^{5}$ & 0 & 1 & 0 & 0 & 20 & 5 & 31 & & 32 \\
\hline$\#=13$ & & $1^{9}$ & 0 & 0 & 0 & 0 & 32 & 95 & 531 & & \\
\hline
\end{tabular}

\begin{tabular}{|c|c|c|c|c|c|c|c|c|}
\hline \multirow[t]{14}{*}{$c_{4}$} & \multirow{14}{*}{$\begin{array}{l}\mathrm{Sp}_{0} \\
9\end{array}$} & \multirow{2}{*}{$\begin{array}{l}\lambda \\
8\end{array}$} & \multicolumn{4}{|c|}{$\leadsto \infty$} & \multirow{2}{*}{$\begin{array}{c}\gamma_{1}(\lambda) \\
0\end{array}$} & \multirow{2}{*}{$\begin{array}{l}\text { ord. } \\
0\end{array}$} \\
\hline & & & 2 & 2 & 2 & 2 & & \\
\hline & & & 2 & 2 & 0 & 2 & 2 & 1 \\
\hline & & $\begin{array}{lll}6 & 1 & 1\end{array}$ & 2 & 2 & 1 & 0 & 4 & 2 \\
\hline & & 44 & 0 & 2 & 0 & 2 & 4 & 11 \\
\hline & & 422 & 2 & 0 & 0 & 2 & 6 & 21 \\
\hline & & 4211 & 2 & 0 & 1 & 0 & 8 & 31 \\
\hline & & $41^{4}$ & 2 & 1 & 0 & 0 & 12 & 42 \\
\hline & & 332 & 0 & 1 & 1 & 0 & 8 & 211 \\
\hline & & $\begin{array}{llll}3 & 3 & 1 & 1\end{array}$ & 0 & 2 & 0 & 0 & 10 & $\begin{array}{lll}3 & 1 & 1\end{array}$ \\
\hline & & 2222 & 0 & 0 & 0 & 2 & 12 & 321 \\
\hline & & $\begin{array}{lllll}2 & 2 & 2 & 1 & 1\end{array}$ & 0 & 0 & 1 & 0 & 14 & 421 \\
\hline & & $221^{4}$ & 0 & 1 & 0 & 0 & 18 & $\begin{array}{lll}5 & 3 & 1\end{array}$ \\
\hline & & $21^{6}$ & 1 & 0 & 0 & 0 & 24 & 642 \\
\hline$\#=14 d$ & & $1^{8}$ & 0 & 0 & 0 & 0 & 32 & 753 \\
\hline
\end{tabular}




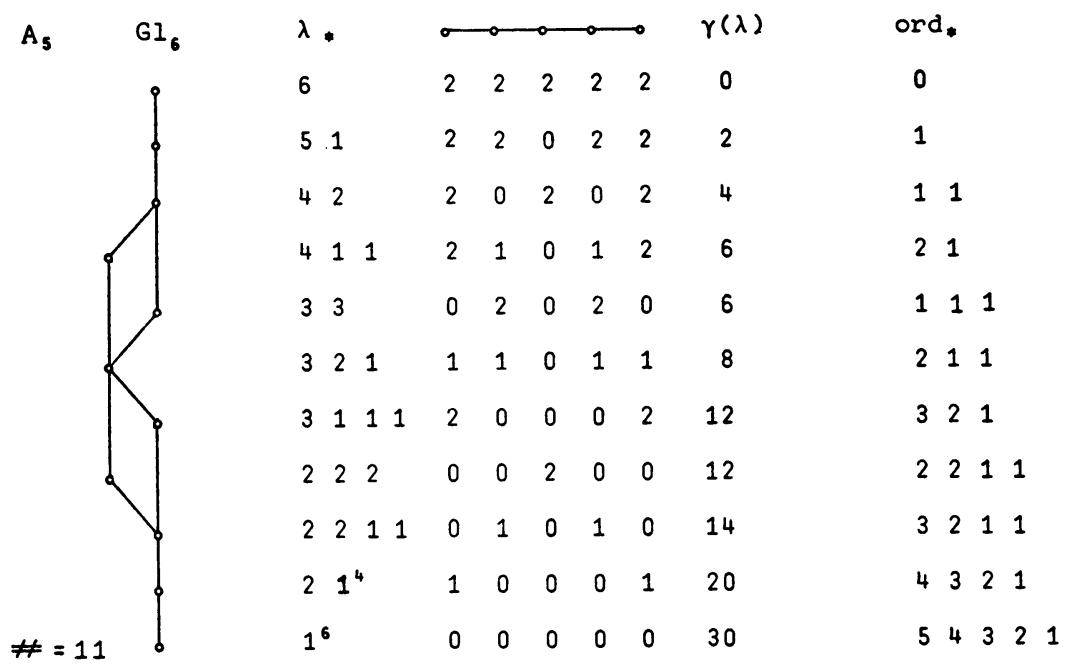

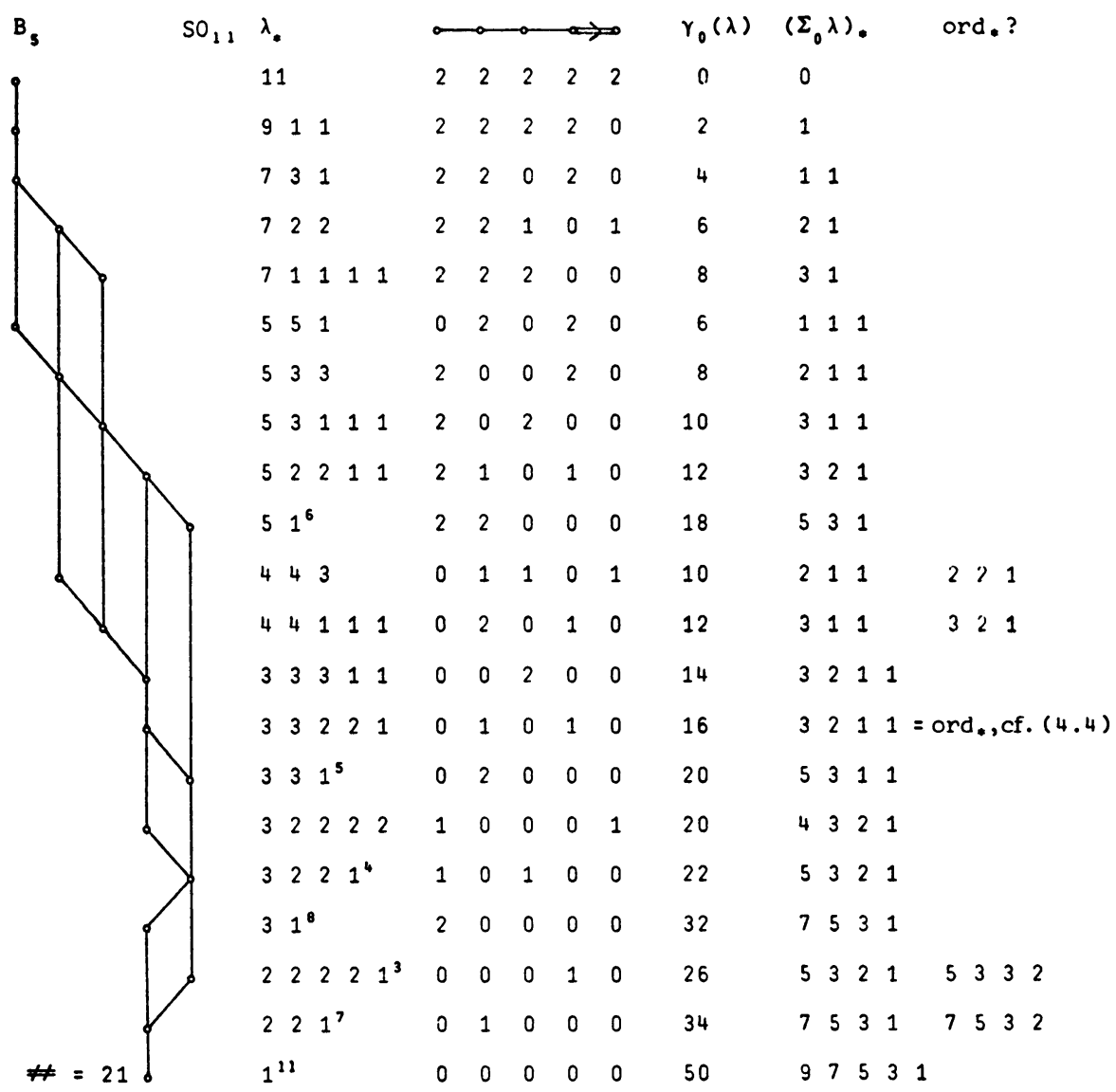




\begin{tabular}{|c|c|c|c|c|c|c|c|c|c|c|c|}
\hline \multirow[t]{24}{*}{$c_{5}$} & \multirow{24}{*}{$\mathrm{Sp}_{10}$} & $\lambda$ & \multicolumn{5}{|c|}{ o } & \multirow{2}{*}{$\begin{array}{c}\gamma_{1}(\lambda) \\
0\end{array}$} & \multicolumn{2}{|c|}{ ord. } & \\
\hline & & 10 & 2 & 2 & 2 & 2 & 2 & & 0 & & \\
\hline & & 82 & 2 & 2 & 2 & 0 & 2 & 2 & 1 & & \\
\hline & & 811 & 2 & 2 & 2 & 1 & 0 & 4 & 2 & & \\
\hline & & 64 & 2 & 0 & 2 & 0 & 2 & 4 & 1 & 1 & \\
\hline & & 622 & 2 & 2 & 0 & 0 & 2 & 6 & 2 & 1 & \\
\hline & & 6211 & 2 & 2 & 0 & 1 & 0 & 8 & 3 & 1 & \\
\hline & & $\begin{array}{lllll}6 & 1 & 1 & 1 & 1\end{array}$ & 2 & 2 & 1 & 0 & 0 & 12 & 4 & 2 & \\
\hline & & 55 & 0 & 2 & 0 & 2 & 0 & 6 & 1 & 11 & \\
\hline & & 442 & 0 & 2 & 0 & 0 & 2 & 8 & 2 & 11 & 1 \\
\hline & & 4411 & 0 & 2 & 0 & 1 & 0 & 10 & 3 & 11 & \\
\hline & & 433 & 1 & 0 & 1 & 1 & 0 & 10 & 2 & 21 & \\
\hline & & 4222 & 2 & 0 & 0 & 0 & 2 & 12 & 3 & 21 & 1 \\
\hline & & $\begin{array}{lllll}4 & 2 & 2 & 1 & 1\end{array}$ & 2 & 0 & 0 & 1 & 0 & 14 & 42 & 21 & 1 \\
\hline & & $421^{4}$ & 2 & 0 & 1 & 0 & 0 & 18 & 5 & 31 & 1 \\
\hline & & $41^{6}$ & 2 & 1 & 0 & 0 & 0 & 24 & 6 & 42 & \\
\hline & & 3322 & 0 & 1 & 0 & 1 & 0 & 14 & 3 & 21 & 1 \\
\hline & & $\begin{array}{lllll}3 & 3 & 2 & 1 & 1\end{array}$ & 0 & 1 & 1 & 0 & 0 & 16 & 4 & 21 & 1 \\
\hline & & $331^{4}$ & 0 & 2 & 0 & 0 & 0 & 20 & 5 & 31 & 1 \\
\hline & & 22222 & 0 & 0 & 0 & 0 & 2 & 20 & 4 & 32 & 1 \\
\hline & & $2^{4} 11$ & 0 & 0 & 0 & 1 & 0 & 22 & 5 & 32 & 1 \\
\hline & & $2^{3} 1^{4}$ & 0 & 0 & 1 & 0 & 0 & 26 & 6 & 42 & 1 \\
\hline & & $221^{6}$ & 0 & 1 & 0 & 0 & 0 & 32 & 7 & 53 & 1 \\
\hline & & $21^{\circ}$ & 1 & 0 & 0 & 0 & 0 & 40 & 8 & 64 & 2 \\
\hline$\#=24$ & & $1^{10}$ & 0 & 0 & 0 & 0 & 0 & 50 & 9 & 75 & 31 \\
\hline
\end{tabular}




\section{WIM HESSELINK}

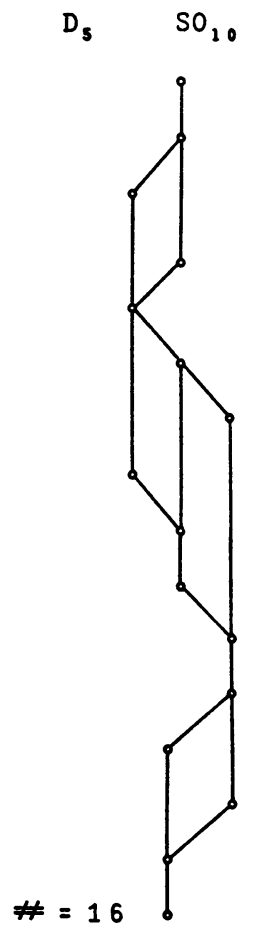

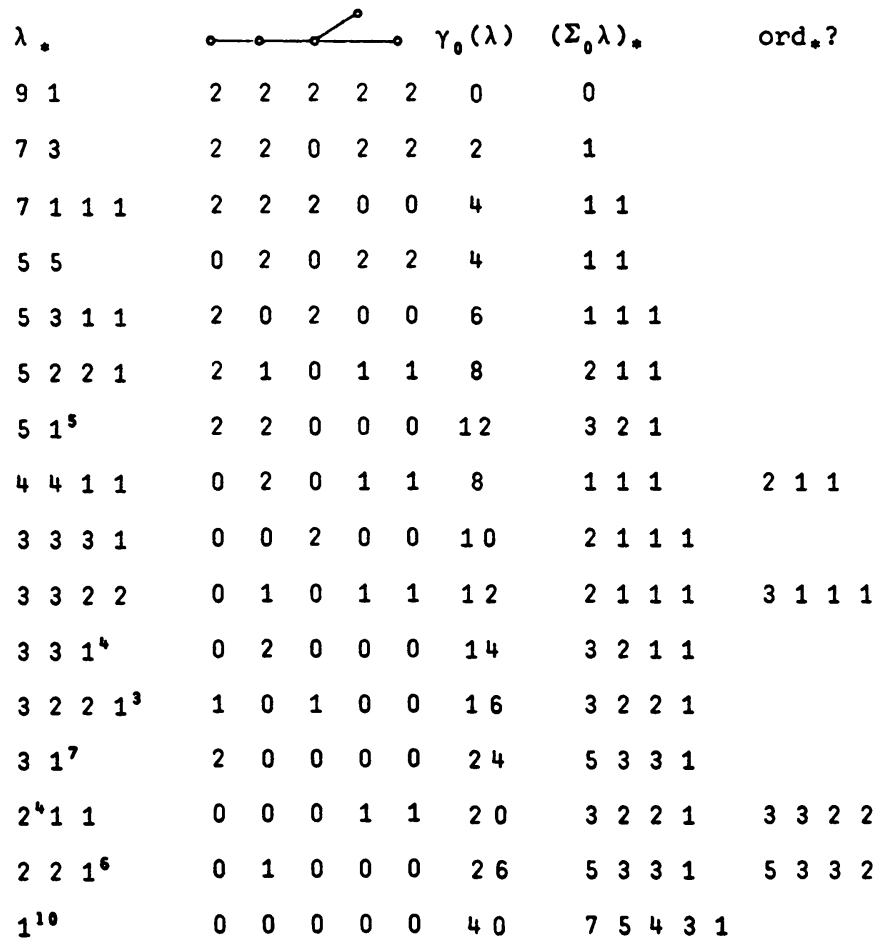

\begin{tabular}{|c|c|c|c|c|c|c|c|c|c|c|}
\hline \multirow[t]{15}{*}{$A_{6}$} & \multirow{15}{*}{$\begin{array}{l}\text { Gl, } \\
9\end{array}$} & \multirow{2}{*}{$\begin{array}{l}\lambda= \\
7\end{array}$} & \multicolumn{6}{|c|}{$a$} & \multirow{2}{*}{$\begin{array}{c}\gamma(\lambda) \\
0\end{array}$} & \multirow{2}{*}{$\begin{array}{l}\text { ord. } \\
0\end{array}$} \\
\hline & & & 2 & 2 & 2 & 2 & 2 & 2 & & \\
\hline & & 61 & 2 & 2 & 1 & 1 & 2 & 2 & 2 & 1 \\
\hline & & 52 & 2 & 1 & 1 & 1 & 1 & 2 & 4 & 11 \\
\hline & & 511 & 2 & 2 & 0 & 0 & 2 & 2 & 6 & 21 \\
\hline & & 43 & 1 & 1 & 1 & 1 & 1 & 1 & 6 & 111 \\
\hline & & 421 & 2 & 0 & 1 & 1 & 0 & 2 & 8 & 211 \\
\hline & & $41^{3}$ & 2 & 1 & 0 & 0 & 1 & 2 & 12 & 321 \\
\hline & & $\begin{array}{lll}3 & 3 & 1\end{array}$ & 0 & 2 & 0 & 0 & 2 & 0 & 10 & 2111 \\
\hline & & 322 & 1 & 0 & 1 & 1 & 0 & 1 & 12 & 2211 \\
\hline & & $\begin{array}{llll}3 & 2 & 1 & 1\end{array}$ & 1 & 1 & 0 & 0 & 1 & 1 & 14 & $\begin{array}{llll}3 & 2 & 1 & 1\end{array}$ \\
\hline & & $31^{4}$ & 2 & 0 & 0 & 0 & 0 & 2 & 20 & 4321 \\
\hline & & 2221 & 0 & 0 & 1 & 1 & 0 & 0 & 18 & $3 /: 11$ \\
\hline & & $221^{3}$ & 0 & 1 & 0 & 0 & 1 & 0 & $2 ?$ & 43711 \\
\hline & & $21^{5}$ & 1 & 0 & 0 & 0 & 0 & 1 & 30 & $r 4321$ \\
\hline 15 & & $1^{7}$ & 0 & 0 & 0 & 0 & 0 & 0 & 42 & 654321 \\
\hline
\end{tabular}




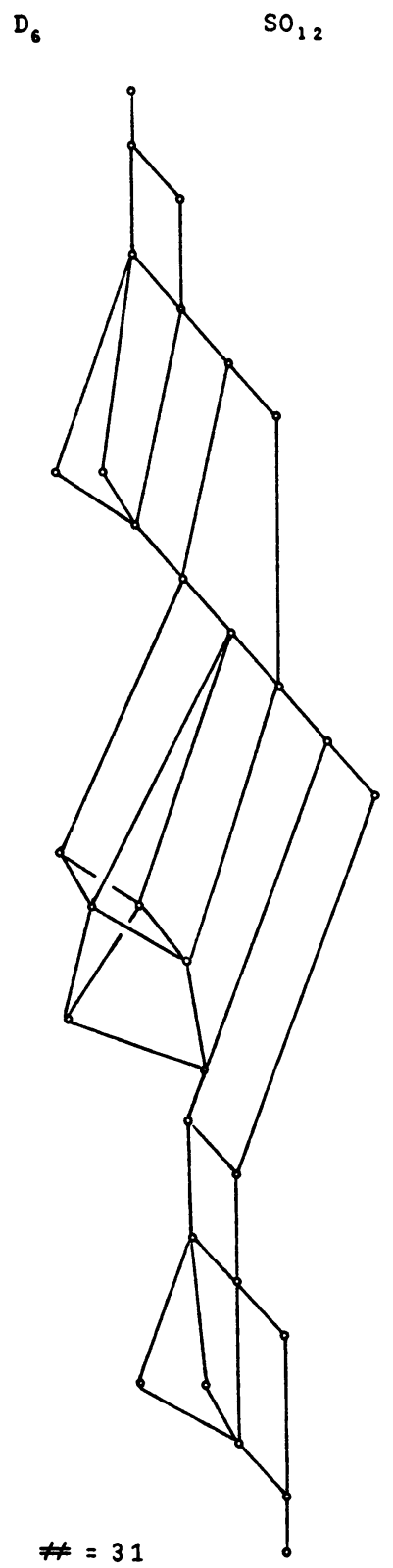

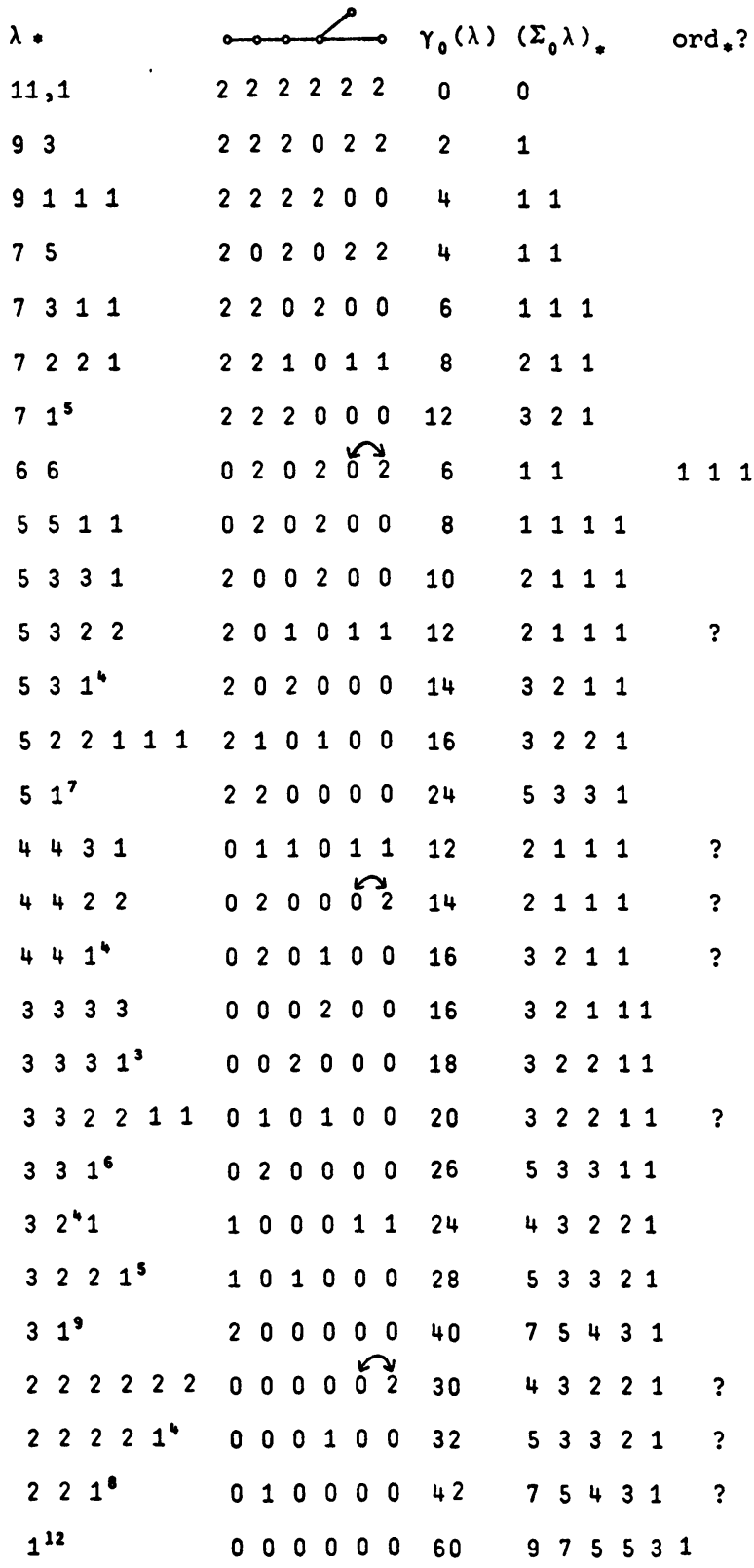




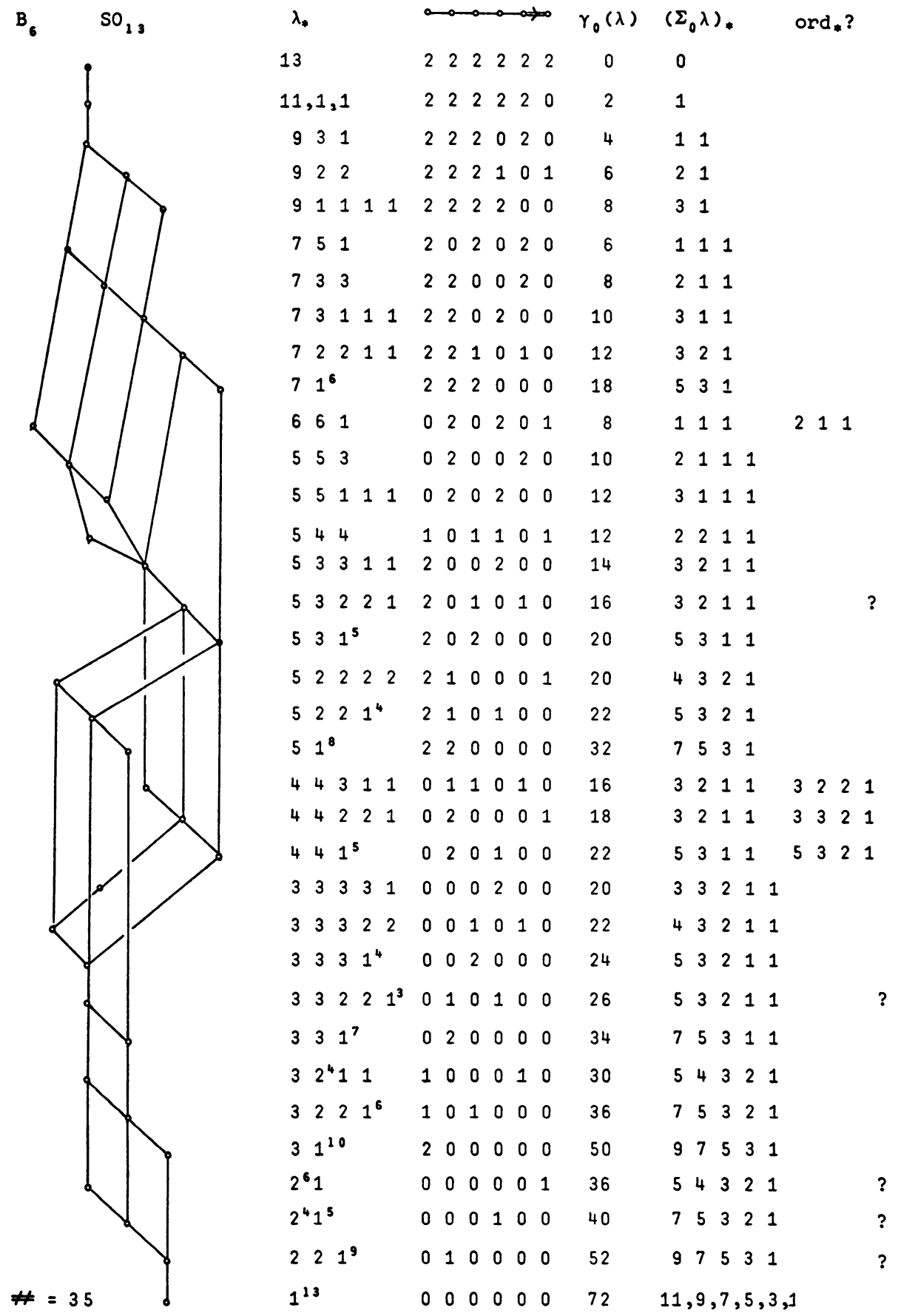




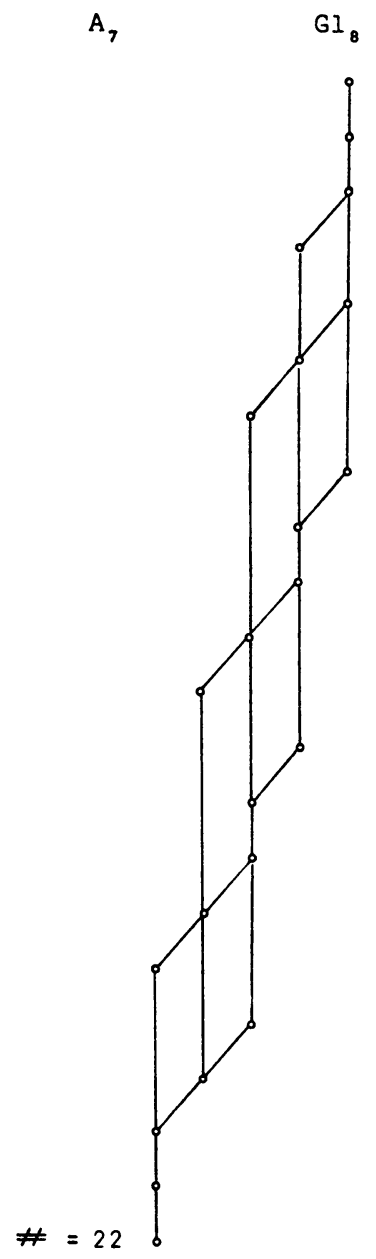

$\begin{array}{llll}\lambda & * \\ 8 & & \\ 7 & 1 & \\ 6 & 2 & \\ 6 & 1 & 1 \\ 5 & 3 & & \\ 5 & 2 & 1 \\ 5 & 1 & 1 & 1 \\ 4 & 4 & \\ 4 & 3 & 1 \\ 4 & 2 & 2 & \\ 4 & 2 & 1 & 1 \\ 4 & 1^{4} & \\ 3 & 3 & 2 \\ 3 & 3 & 1 & 1 \\ 3 & 2 & 2 & 1 \\ 3 & 2 & 1^{3} \\ 3 & 1^{5} & \\ 2 & 2 & 2 & 2 \\ 2 & 2 & 2 & 1 \\ 2 & 2 & 1^{4} \\ 2 & 1^{6} & \\ 1^{8} & & \\ \end{array}$

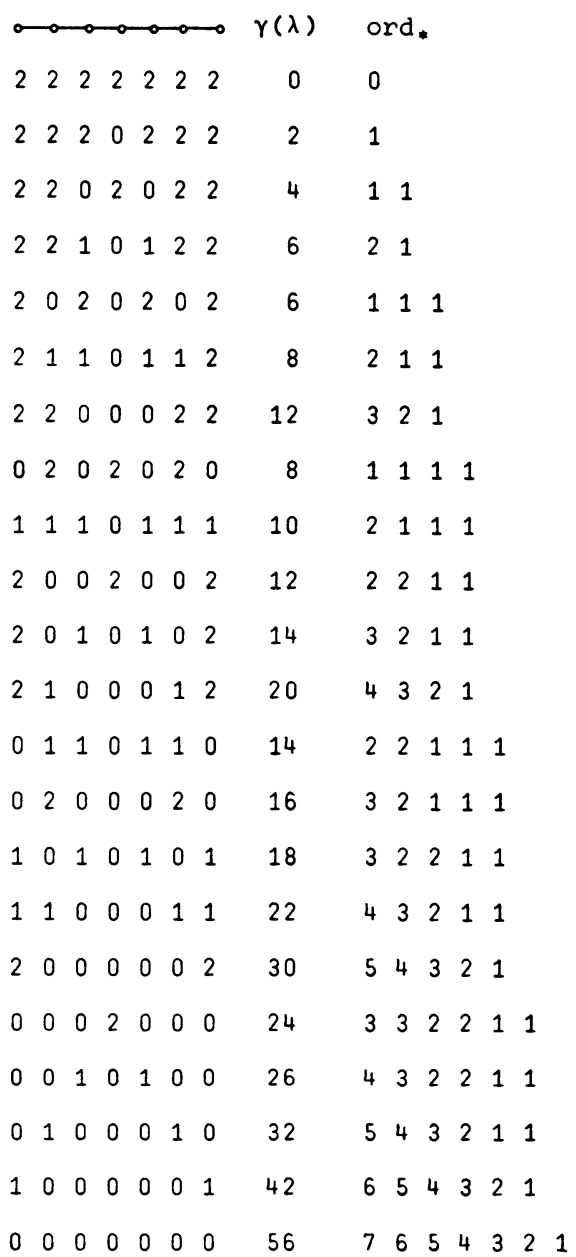

\section{REFERENCES}

1. V. I. Arnol'd, On matrices depending on parameters, Uspehi Mat. Nauk 26 (1971), no. 2 (158), $101-114$ = Russian Math. Surveys 26 (1971), no. 2, 29-43. MR 46 \#400.

2. A. Borel, Linear algebraic groups, Benjamin, New York, 1969. MR 40 \#4273.

3. N. Bourbaki, Eléments de mathématique. Les structures fondamentales de l'analyse. Livre II: Algèbre, Chap. 9: Formes sesquilinéaires et formes quadratiques, Actualités Sci. Indust., no. 1272, Hermann, Paris, 1959. MR 21 \#6384.

4. - Eléments de mathématique. Fasc. XXXIV. Groupes et algèbres de Lie. Chaps. IV, V, VI, Actualités Sci. Indust., no. 1337, Hermann, Paris, 1968. MR $39 \#_{1} 590$.

5. E. Brieskorn, Singular elements of semisimple algebraic groups, Proc. Internat.

Congress Math. (Nice, 1970), vol. II, Gauthier-Villars, Paris, 1971, pp. 279-284.

6. C. Chevalley, Classification des groupes de Lie algébriques, Notes polycopiées, Inst. H. Poincaré, Paris, 1956-1958.

7. M. Demazure, Invariants symétriques entiers des groupes de Weyl et torsion, Invent. Math. 21 (1973), 287-301. MR 49 \#268.

8. M. Demazure and A. Grothendieck, Schémas en groupes (SGA 3), Lecture Notes in Math., vols. 151, 152, 153, Springer-Verlag, Berlin, 1970.

9. M. Gerstenhaber, On dominance and varieties of commuting matrices, Ann. of Math. (2) 73 (1961), 324-348. MR 24 \#A1926. 
10. M. Gerstenhaber, Dominance over the classical groups, Ann. of Math. (2) 74 (1961), 532-569. MR 25 \#148.

11. A. Grothendieck and J. A. Dieudonné, Eléments de géométrie-algébrique. I, Die Grundlehren der math. Wissenschaften, Band 166, Springer-Verlag, Berlin, 1971.

12. - Eléments de géométrie algébrique. II, III, IV, Inst. Hautes Études Sci. Publ. Math. Nos. 8, 11, 17, 20, 24, 28, 32 (1961-1967). MR 29 \#1212; 30 \#3885; 33 \#7330; 36 \#177b, c; \#178; 39 \#220.

13. W. H. Hesselink, Singularities in the nilpotent scheme of a classical group. Thesis, Utrecht, 1975.

14. H. Hironaka, Resolution of singularities of an algebraic variety over a field of characteristic zero. I, II, Ann. of Math. (2) 79 (1964), 109-326. MR 33 \#7333.

15. B. Iversen, Generic local structure in commutative algebra, Lecture Notes in Math., vol. 310, Springer-Verlag, Berlin, 1973.

16. B. Kostant, Lie group representations on polynomial rings, Amer. J. Math. 85 (1963), 327-404. MR 28 \#1252.

17. D. Mumford, Geometric invariant theory, Ergebnisse der Mathematik und ihrer Grenzgebiete, Band 34, Springer-Verlag, Berlin; Academic Press, New York, 1965. MR 35 \#5451.

18. J.-J. Risler, Algèbre, symétrique d'un ideal, Sur Quelques Problèmes d'Algèbre, Univ. de Montpellier, Montpellier, 1969, pp. 61-69. MR 40 \#253.

19. T. A. Springer, The unipotent variety of a semi-simple group, Algebraic Geometry (Internat. Colloq., Tata Inst. Fund. Res., Bombay, 1968), Oxford Univ. Press, London, 1969, pp. 373-391. MR 41 \#8429.

20. T. A. Springer and R. Steinberg, Conjugacy classes, Seminar on Algebraic Groups and Related Finite Groups (The Institute for Advanced Study, Princeton, N. J., 1968/69), Lecture Notes in Math., vol. 131, Springer-Verlag, Berlin, 1970, pp. 167-266. MR 42 \#3091.

21. R. Steinberg, Conjugacy classes in algebraic groups, Lecture Notes in Math., vol. 366, Springer-Verlag, Berlin, 1974.

22. F. D. Veldkamp, The center of the universal enveloping algebra of a Lie algebra in characteristic p, Ann. Sci. Ecole Norm. Sup. (4) 5 (1972), 217-240.

MATHEMATISCH INSTITUUT DER RIJKSUNIVERSITEIT TE UTRECHT, UTRECHT, THE NETHERLANDS

Current address: Mathematisch Instituut, Postbus 800, Groningen, The Netherlands 